\title{
Potential nutrient-response curves and sufficiency ranges of 'Grand Nain' banana cultivated in two environment
}

\author{
Vagner Alves Rodrigues Filho ${ }^{1}$, Júlio César Lima Neves², \\ Sérgio Luiz Rodrigues Donato ${ }^{3}$, Bruno Vinícius Castro Guimarães ${ }^{4}$
}

\begin{abstract}
Adequate plant nutrition is essential to attain higher yields. The objective was to determine potential-nutrient response curves and sufficiency ranges using the boundary line approach and balance indices of Kenworthy for interpreting the nutritional status of 'Grand Nain' banana cultivated in two environments. The study was carried out using a database containing leaf nutrient concentrations and yields of bananas cultivated on two areas located in Missão Velha-CE, and Ponto Novo-BA, Brazil. Plots with high-yielding plants, which were those with yields above average plus 0.5 standard deviation, were used atem as reference population. The database was subdivided into two sets. One of them contained 46 leaf tissue samples and reference population with yield greater than $58.84 \mathrm{Mg} \mathrm{ha}^{-1}$ year $^{-1}$, in Missão Velha-CE. The second data set contained 19 samples and reference population with yield greater than $76.12 \mathrm{Mg} \mathrm{ha}^{-1} \mathrm{year}^{-1}$ in Ponto Novo-BA. Potential response curves were fitted to the relationship between relative yield and leaf element concentrations and balance indices of Kenworthy. Models expressed high predictive power. Sufficiency ranges for macro- and micronutrient concentrations and balance indices of Kenworthy were established. The ranges allow an improved nutritional status assessment of irrigated 'Grand Nain' bananas.

Index terms: Musa spp. AAA, boundary line, diagnostic methods, nutritional status.
\end{abstract}

\section{Curvas de resposta potencial e faixas de suficiência de nutrientes para bananeira 'Grande Naine' em dois ambientes}

Corresponding author: bvinicius20@yahoo.com.br

Received: October 09, 2020 Accepted: January 04, 2021

Copyright: All the contents of this journal, except where otherwise noted, is licensed under a Creative Commons Attribution License.
Resumo - A nutrição adequada das plantas é essencial para obter maiores rendimentos. O objetivo foi determinar curvas de resposta potencial de nutrientes e intervalos de suficiência utilizando a abordagem da linha de fronteira e índices de equilíbrio de Kenworthy para interpretar o estado nutricional da bananeira 'Grande-Naine' cultivada em dois ambientes. O estudo foi realizado em um banco de dados contendo as concentrações foliares de nutrientes e as produtividades da bananeiras cultivadas em duas áreas de produção localizadas em Missão Velha-CE, e Ponto Novo-BA, Brasil. Parcelas com plantas de alto rendimento, correspondentes às produtividades acima da média $+/-0,5$ desvio padrão, foram utilizadas como população de referência. $\mathrm{O}$ banco de dados foi subdividido em dois conjuntos. Um deles continha 46 amostras de tecido foliar e população de referência com produtividade superior a 58,84 Mg ha ${ }^{-1}$ ano $^{-1}$, em Missão Velha-CE. O segundo conjunto de dados continha 19 amostras e população de referência com produtividade superior a $76,12 \mathrm{Mg} \mathrm{ha}^{-1}$ ano $^{-1} \mathrm{em}^{-}$ Ponto Novo-BA. As curvas de resposta potencial foram ajustadas entre o rendimento relativo com as concentrações de elementos na folha, e com os índices de equilíbrio de Kenworthy. Os modelos expressaram alto poder preditivo. Foram estabelecidas faixas de suficiência para concentrações de macro e micronutrientes, e índices de equilíbrio de Kenworthy. Os intervalos permitem uma avaliação eficiente do estado nutricional de bananeiras 'Grande Naine' irrigadas.

Termos para indexação: Musa spp. AAA, linha de fronteira, métodos de diagnóstico, estado nutricional.

\footnotetext{
${ }^{1}$ Agronomist MSc in Phytotechnics, Technical Sector, Sítio Barreiras Fruticultura Ltda. - Fazenda Barreiras - Missão Velha-CE, Brazil. E-mail: vagner_loiola@yahoo.com.br(ORCID 0000-0001-7702-7445)

²DSc in Crop Production, Professor at the Federal University of Viçosa, Soil Department. Viçosa-MG, Brazil. Email: julio_n2003@yahoo. com.br (ORCID 0000-0001-8356-5100)

${ }^{3} \mathrm{DSc}$ in Phytotechnics, Professor at the Federal Institute of Education, Science and Technology Baiano, Campus Guanambi, Agriculture Sector, Guanambi-BA, Brazil. E-mail: sergio.donato@ifbaiano.edu.br (ORCID 0000-0002-7719-4662)

${ }^{4} \mathrm{DSc}$ in Phytotechnics, Professor at the Federal Institute of Education, Science and Technology of Amazonas, Campus São Gabriel da Cachoeira, São Gabriel da Cachoeira-AM, Brazil. E-mail: bvinicius20@yahoo.com.br ${ }^{(O R C I D}$ 0000-0003-2585-8794)
} 


\section{Introduction}

Assessing the nutritional status of plants is required when increasing crop production while lessening environmental degradation (ALI, 2018). Sufficiency ranges have been used as a traditional tool in plant nutritional diagnosis; however, the method is statistic based and thus prone to interpreting mistakes due to variable element concentrations in leaves. This approach has been further criticized for not taking into consideration interactions between nutrients (BARKER; PILBEAM, 2007; MARSCHNER, 2012). Nonetheless, Quaggio and Raij (1997) reported sufficiency ranges for Cavendish bananas grown in the state of São Paulo, and Pauletti and Motta (2017) for in the state of Paraná, in Brazil.

Nutrient standards are conventionally established in calibration tests in which genetic and environmental factors and interactions between nutrients are controlled (WADT et al., 1998). As element concentrations in plant tissues are influenced by several environmental and crop-related factors, wide-ranging sufficiency ranges established under varying environmental conditions may lead to inaccurate diagnoses. Iheshiulo et al. (2019) pointed out that methods that determine region-specific nutrient reference values may provide more accurate results; therefore, establishing region- and cultivar-specific sufficiency ranges can be significantly more advantageous.

The boundary line approach is an alternative to calibration tests as it uses nutritional data from commercial crop fields. This approach consists of plotting leaf nutrient concentrations against yield observations, so that the optimum leaf concentration for a given nutrient or nutrient ratios is determined based on data points on the edge of the scatter plot (boundary line). Moreover, it allows the estimation of the maximum attainable yield (ALMEIDA et al., 2016; ALI, 2018).

Boundary-line sufficiency ranges have been reported for several crops including forage cactus (BLANCOMACÍAS et al., 2010), wild blueberry (LAFOND, 2009), papaya (MAIA; MORAIS, 2016), sugarcane
(MCCRAYET al., 2010), rubber tree (NJUKENG et al. 2013), pitaya (ALMEIDA et al., 2016), and eucalyptus (LIMA NETO et al., 2020); however, studies on bananas grown under different conditions are in demand.

The objective of this study was to establish potential response curves and sufficiency ranges by employing the boundary line approach and balance indices of Kenworthy to interpret the nutritional status of 'Grand Nain' bananas cultivated in two production conditions.

\section{Material and methods}

The data used in this study were from two farms belonging to the company Sítio Barreiras. The first farm is located in the municipality of Missão Velha, state of Ceará (CE), Brazil ( $7^{\circ} 35^{\prime} 9^{\prime}$ ' S, 39 $21^{\circ} 1^{\prime}$ ' $\mathrm{W}$, and altitude of 442 $\mathrm{m})$. The climate of the region is Aw - tropical savanna climate with dry winters and rainy summers (KöppenGeiger). Mean annual rainfall and temperature are 942 $\mathrm{mm}$ and $25.8^{\circ} \mathrm{C}$, respectively. The soil at the farm was, predominately, a Latossolo Vermelho-Amarelo (SANTOS et al., 2018), which corresponds to Oxisol (SOIL SURVEY STAFF, 2014). On this farm, the company has 11 plots, each measuring 3.26 ha, where fertigated 'Grand Nain' bananas are cultivated.

The second farm is located in the municipality of Ponto Novo, state of Bahia (BA), Brazil, (10 $10^{\circ}$ '46” S, $40^{\circ} 08^{\prime} 01^{\prime \prime} \mathrm{W}$, and altitude of $342 \mathrm{~m}$ ). The climate is also Aw, according to Köppen-Geiger classification. Mean annual rainfall and temperature are $697 \mathrm{~mm}$ and $24.1^{\circ} \mathrm{C}$, respectively. The predominant soil has been classified as a Latossolo Amarelo (SANTOS et al., 2018), which corresponds to Oxisol (SOIL SURVEY STAFF, 2014). On this farm, the company has 17 plots, each measuring 4.53 ha in average, where fertigated 'Grand Nain' bananas are cultivated.

Chemical properties of the soils and meteorological data from both sites are in Table 1 and 2, respectively. Leaf tissue analysis results and yields recorded in a database were used in this study.

Table 1. Chemical properties of the soils from 'Grand Nain' fields in Missão Velha, CE, and Ponto Novo, BA, Brazil, $0.00-0.20 \mathrm{~m}$ and $0.20-0.40 \mathrm{~m}$.

\begin{tabular}{|c|c|c|c|c|c|c|c|c|c|c|}
\hline \multirow[b]{2}{*}{ Area } & \multirow[b]{2}{*}{ Layers (m) } & \multirow[b]{2}{*}{$\mathrm{pH}$} & $\mathrm{OM}$ & $\mathrm{P}$ & $\mathrm{K}^{+}$ & $\mathrm{Ca}^{++}$ & $\mathrm{Mg}^{++}$ & $\mathrm{CEC}$ & \multirow{2}{*}{$\frac{\mathrm{V}}{\%}$} & \multirow{2}{*}{$\frac{\text { P-Rem }}{\mathrm{mg} \mathrm{L}^{-1}}$} \\
\hline & & & $\mathrm{g} \mathrm{dm}^{-3}$ & $\mathrm{mg} \mathrm{dm}{ }^{-3}$ & \multicolumn{4}{|c|}{---------mmol $\mathrm{dm}^{-3}$------ } & & \\
\hline Missão & $0.00-0.20$ & 7.4 & 32.0 & 140.0 & 9.3 & 113.0 & 27.0 & 156.0 & 95.0 & 54.1 \\
\hline Velha & $0.20-0.40$ & 7.9 & 18.0 & 79.0 & 4.9 & 91.0 & 21.0 & 124.0 & 94.0 & 47.9 \\
\hline Ponto & $0.00-0.20$ & 6.5 & 18.0 & 81.0 & 3.8 & 27.0 & 10.0 & 52.0 & 80.0 & 44.7 \\
\hline Novo & $0.20-0.40$ & 6.1 & 12.0 & 28.0 & 2.4 & 14.0 & 5.0 & 32.0 & 62.0 & 43.5 \\
\hline
\end{tabular}

$\mathrm{pH}$ in water in a 1:2.5 ratio; OM: soil organic matter content obtained by organic carbon $\times 1.724$ (Walkley-Black); P and $\mathrm{K}^{+}$: Mehlich-1 extraction; $\mathrm{Ca}^{2+}$ and $\mathrm{Mg}^{2+}=\mathrm{KCl} 1 \mathrm{~mol} \mathrm{~L}{ }^{-1}$; CEC: cation exchange capacity at $\mathrm{pH} 7$; V: base saturation; P-rem: P-remaining.

Source: elaborate by authors chemical properties of the soils Database of Farms located in Missão Velha, CE, and Ponto Novo, BA. 
Table 2. Meteorological data recorded on the farms located in Missão Velha, CE, and Ponto Novo, BA, Brazil, in 2016.

\begin{tabular}{|c|c|c|c|c|c|c|c|}
\hline \multirow{2}{*}{ Month } & $\begin{array}{c}\text { Mean } \\
\text { temperature }\end{array}$ & $\begin{array}{c}\text { Max. } \\
\text { temperature }\end{array}$ & $\begin{array}{c}\text { Min. } \\
\text { temperature }\end{array}$ & Rainfall & RH & VPD & $\begin{array}{l}\text { Max. wind } \\
\text { speed }\end{array}$ \\
\hline & & $\left({ }^{\circ} \mathrm{C}\right)$ & & $(\mathrm{mm})$ & $(\%)$ & $(\mathrm{kPa})$ & $\left(\mathrm{m} \mathrm{s}^{-1}\right)$ \\
\hline \multicolumn{8}{|c|}{ Missão Velha - CE } \\
\hline January & 26.91 & 31.96 & 21.86 & 231.10 & 74.22 & 0.76 & 1.60 \\
\hline February & 26.95 & 33.17 & 20.73 & 60.90 & 77.61 & 0.67 & 1.60 \\
\hline March & 27.79 & 33.38 & 22.21 & 198.50 & 78.13 & 0.68 & 1.54 \\
\hline April & 27.05 & 32.84 & 21.26 & 33.50 & 74.05 & 0.78 & 3.09 \\
\hline May & 27.14 & 33.40 & 20.88 & 30.00 & 66.65 & 1.01 & 3.09 \\
\hline June & 26.23 & 32.64 & 19.82 & 17.60 & 64.00 & 1.05 & 7.72 \\
\hline July & 26.39 & 33.22 & 19.57 & 0.00 & 50.60 & 1.46 & 5.14 \\
\hline August & 27.00 & 34.68 & 19.32 & 0.00 & 45.92 & 1.67 & 5.14 \\
\hline September & 28.29 & 35.58 & 21.01 & 3.10 & 45.66 & 1.78 & 4.63 \\
\hline October & 29.26 & 36.72 & 21.81 & 0.00 & 44.07 & 1.93 & 3.60 \\
\hline November & 29.67 & 36.32 & 23.03 & 0.00 & 43.41 & 1.97 & 3.09 \\
\hline December & 29.04 & 35.61 & 22.47 & 69.10 & 52.98 & 1.58 & 3.09 \\
\hline \multicolumn{8}{|c|}{ Ponto Novo - BA } \\
\hline January & 25.19 & 29.84 & 22.37 & 190.83 & 82.92 & 0.48 & 5.18 \\
\hline February & 25.83 & 31.86 & 21.08 & 20.80 & 74.06 & 0.75 & 5.98 \\
\hline March & 26.90 & 32.95 & 21.85 & 0.00 & 69.02 & 0.94 & 6.58 \\
\hline April & 26.51 & 32.63 & 21.37 & 14.45 & 64.73 & 1.05 & 5.58 \\
\hline May & 24.48 & 29.58 & 20.45 & 49.25 & 76.05 & 0.64 & 6.21 \\
\hline June & 23.12 & 27.93 & 19.47 & 31.55 & 78.08 & 0.54 & 5.68 \\
\hline July & 22.60 & 28.29 & 18.16 & 8.85 & 75.48 & 0.60 & 6.11 \\
\hline August & 23.33 & 29.25 & 18.57 & 11.75 & 71.71 & 0.72 & 6.50 \\
\hline September & 24.48 & 30.63 & 19.66 & 1.80 & 69.65 & 0.82 & 6.75 \\
\hline October & 25.99 & 32.57 & 20.86 & 5.95 & 66.99 & 0.98 & 7.10 \\
\hline November & 24.53 & 25.23 & 23.82 & 184.00 & 70.79 & 0.74 & 1.34 \\
\hline December & 25.20 & 25.98 & 24.42 & 44.20 & 69.49 & 0.80 & 1.32 \\
\hline
\end{tabular}

RH: relative humidity; VPD: vapor pressure deficit. Source: made by authors from meteorological data recorded by automatic weather stations installed at farms located in Missão Velha-CE, and Ponto Novo-BA, Brazil.

Leaf tissue sampling followed recommendations of Martin-Prével (1987), modified by Rodrigues et al. (2010). The mid-portion of the third leaf lamina, counting from the plant apex, was collected when the inflorescence had two or three open clusters of male flowers. Samples were processed and stored as leaf macro- $(\mathrm{N}, \mathrm{P}, \mathrm{K}, \mathrm{Ca}$, $\mathrm{Mg}$ and $\mathrm{S}$ ) and micronutrient $(\mathrm{B}, \mathrm{Cu}, \mathrm{Fe}, \mathrm{Mn}$ and $\mathrm{Zn})$ concentrations (BATAGLIA et al., 1983).

Yields were estimated as $\mathrm{Mg} \mathrm{ha}^{-1}$ year $^{-1}$ by weighing all bunches in the plot. Leaf analyses were carried out once a semester, with samples collected in summer and in winter.

The database was divided into two site-specific databases. One of them was from the farm located in Missão Velha, CE. It contained tissue analysis results from samples collected twice a year, and annual yields recorded between 2010 and 2017 from 'Grand Nain' banana (AAA) plantations. The initial sample containing 150 recordings, mean \pm standard deviation of $52.35 \pm 12.98 \mathrm{t} \mathrm{ha}^{-1}$ year $^{-1}$, was subdivided into low- and high-yielding populations. High-yielding populations were considered as reference populations and had yields greater than mean +0.5 standard deviation, which corresponded to $58.84 \mathrm{tha}^{-1}$ year- $^{-1}$ for a sample size of 46 .

The other database was from the farm located in Ponto Novo, BA, and contained tissue analysis results from samples collected twice a year and annual yields recorded between 2014 and 2016. The initial sample consisting of 65 recordings, mean \pm standard deviation of $65.15 \pm 21.94 \mathrm{Mg} \mathrm{ha}^{-1}$ year $^{-1}$, was also subdivided into lowand high-yielding populations. The reference population was that with yield greater than mean +0.5 standard deviation, which corresponded to $76.12 \mathrm{Mg} \mathrm{ha}^{-1}$ year $^{-1}$, for a sample size of 19 .

Nutrient sufficiency ranges were determined by an equation adjusted as a function of the relationship between relative yields (RY) on the $y$-axis and leaf nutrient concentrations on the $\mathrm{x}$-axis, forming a scatter plot. Data points located on the edge of the scatter plot were selected using the computer program "Boundary Fit" as used Almeida et al. (2016). 
After identifying the points representing the highyielding plots located on the edge of the scatter data (boundary line), corresponding regression models were created using the application Curve Expert Basic 1.4. Relative yield (\%) was the dependent variable while leaf nutrient concentration, the independent variable.

With the boundary line, sufficiency ranges of each nutrient were determined based on the following interpretative categories: deficient $(\mathrm{RY}<70 \%)$, tendency towards sufficient $(70 \leq \mathrm{RY}<90 \%)$, sufficient $(90 \leq \mathrm{RY}$ $<100 \%$ ), high (100 $>$ RY $\geq 90 \%$, right to the maximum), tendency towards excessive $(90 \leq \mathrm{RY}<70 \%$, right to the maximum), and excessive ( $\mathrm{RY}<70 \%$, right to the maximum). These ranges are proposed because the original method was established for apple trees.
The mean and variability of leaf nutrient concentrations were obtained from the reference population (Table 3). Standard-index or percent of the standard (P) (ratio of sample nutrient concentration to standard concentration), influence of variation (I) and coefficient of variation (CV) were calculated. The results were expressed as percentage (URANO et al., 2007).

Table 3. Norms used to determine balance indices of Kenworthy for 'Grand Nain' bananas grown in Missão VelhaCE, and Ponto Novo-BA, Brazil.

\begin{tabular}{lccccccc}
\hline & \multicolumn{3}{c}{ Missão Velha - Ceará } & \multicolumn{5}{c}{ Ponto Novo - Bahia } \\
\hline Norm & $\bar{Y}$ & $\mathrm{~S}$ & $\mathrm{CV}(\%)$ & $\mathrm{Norm}$ & $\overline{\mathrm{Y}}$ & $\mathrm{S}$ & $\mathrm{CV}(\%)$ \\
\hline $\mathrm{N}$ & 21.7667 & 1.8413 & 8.46 & $\mathrm{~N}$ & 22.0211 & 1.8504 & 8.40 \\
$\mathrm{P}$ & 1.6368 & 0.2274 & 13.89 & $\mathrm{P}$ & 1.5632 & 0.1116 & 7.14 \\
$\mathrm{~K}$ & 36.1928 & 6.3267 & 17.48 & $\mathrm{~K}$ & 32.6421 & 7.7467 & 23.73 \\
$\mathrm{Ca}$ & 7.8817 & 1.9026 & 24.14 & $\mathrm{Ca}$ & 6.9737 & 0.8621 & 12.36 \\
$\mathrm{Mg}$ & 2.5923 & 0.3945 & 15.22 & $\mathrm{Mg}$ & 2.8526 & 0.1264 & 4.43 \\
$\mathrm{~S}$ & 1.5441 & 0.2229 & 14.44 & $\mathrm{~S}$ & 1.4947 & 0.1177 & 7.88 \\
$\mathrm{~B}$ & 9.6391 & 3.0533 & 31.68 & $\mathrm{~B}$ & 13.4053 & 2.1441 & 15.99 \\
$\mathrm{Cu}$ & 5.9109 & 2.6298 & 44.49 & $\mathrm{Cu}$ & 6.4947 & 0.5921 & 9.12 \\
$\mathrm{Fe}$ & 68.7087 & 10.9113 & 15.88 & $\mathrm{Fe}$ & 59.0211 & 9.1099 & 15.43 \\
$\mathrm{Mn}$ & 168.8761 & 121.8828 & 72.17 & $\mathrm{Mn}$ & 73.5474 & 16.8846 & 22.96 \\
$\mathrm{Zn}$ & 15.7891 & 2.8883 & 18.29 & $\mathrm{Zn}$ & 15.3895 & 1.3316 & 8.65 \\
\hline
\end{tabular}

Norm $=$ reference population concentrations $\left(\geq\right.$ mean +0.5 standard deviation), $\mathrm{N}, \mathrm{P}, \mathrm{K}, \mathrm{Ca}, \mathrm{Mg}$ and $\mathrm{S}$ contents are expressed as $\mathrm{g} \mathrm{kg}{ }^{-1}$, and $\mathrm{Cu}$, $\mathrm{Fe}, \mathrm{Zn}, \mathrm{Mn}$ and $\mathrm{B}$, as $\mathrm{mg} \mathrm{kg}^{-1} ; \mathrm{Y}=$ mean nutrient content in leaf sample; $\mathrm{s}=$ standard deviation; $\mathrm{CV}=$ coefficient of variation.

The following equations were used to calculate balance indices:

$$
\begin{aligned}
& \mathrm{P}=(100 \mathrm{yi}) / \hat{\mathrm{Y}} \\
& \mathrm{I}=\mathrm{CV}(\mathrm{yi}-\hat{\mathrm{Y}}) / \hat{\mathrm{Y}} \\
& \mathrm{BIKW}=\mathrm{P}-1
\end{aligned}
$$

where, $\mathrm{P}=$ ratio of sample nutrient concentration (yi) to standard concentration $(\bar{Y})(\%)$; $\mathrm{I}=$ influence of variation (\%); $\mathrm{CV}=$ coefficient of variation (\%) of nutrient concentrations in the reference population; BIKW = balance index of Kenworthy.

BIKM method establishes the upper and lower limits of each sufficiency range. The upper limit of the sufficient range is the concentration that corresponds to $100 \%$.
Sufficiency ranges of each nutrient were determined taking into account limits proposed by Kenworthy (1961): deficient (BIKW $<50 \%$ ); tendency towards sufficient (50 $\leq$ BIKW $<83 \%$ ); sufficient ( $83 \leq$ BIKW $<100 \%$ ); high $(100 \leq$ BIKW $<117 \%)$; tendency towards excessive $(117$ $\leq$ BIKW $<150 \%)$; and excessive (BIKW $\geq 150 \%$ ).

After obtaining the balance indices of each nutrient based on site-specific norms, these indices were plotted against relative yields. The boundary line approach was then used as previously described; therefore, new concentration ranges of each nutrient were generated based on the following interpreting categories: deficient ( $\mathrm{RY}<70 \%$ ), tendency towards sufficient $(70 \leq \mathrm{RY}<90 \%)$, sufficient $(90 \leq \mathrm{RY}<100 \%)$, high $(100>\mathrm{RY} \geq 90 \%$, right to the maximum), tendency towards excessive $(90 \leq \mathrm{RY}<$ $70 \%$, right to the maximum); and excessive (RY $<70 \%$, right to the maximum). 


\section{Results and Discussion}

Using yields observations and leaf nutrient concentrations of 'Grand Nain' bananas grown in the states of Ceará and Bahia, boundary lines (Figures 1 and 2) were fitted to the relationship between relative yields

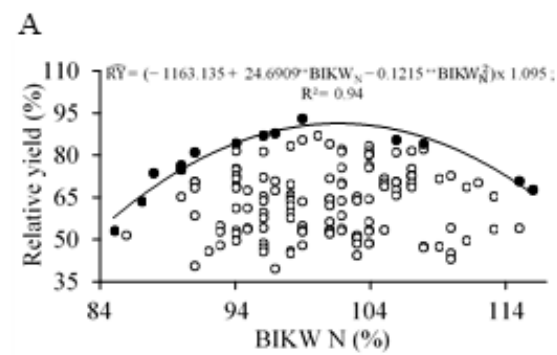

C

E

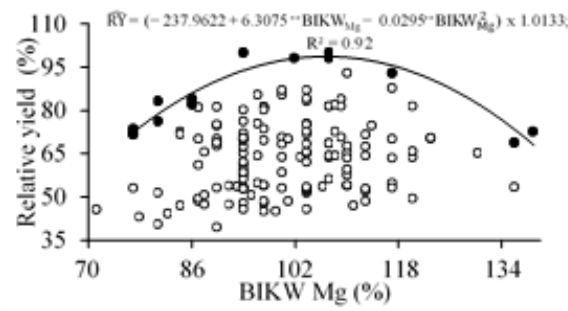

G

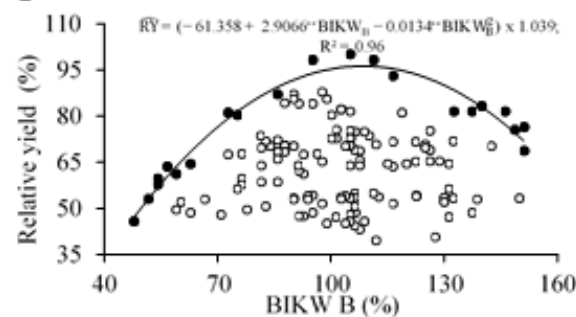

I

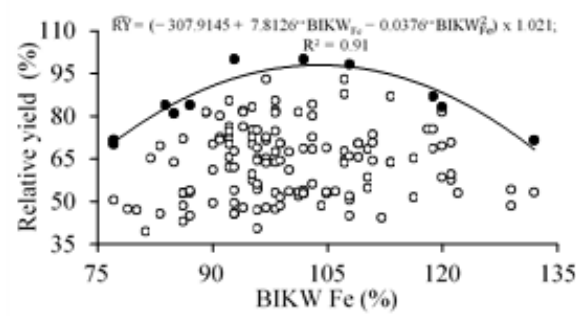

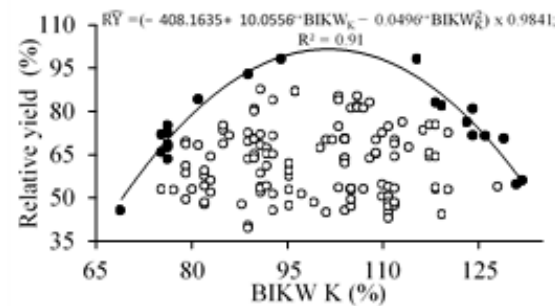

and balance indices of Kenworthy (BIKW). Regression equations were adjusted to represent the boundary lines. The models were quadratic, significant, and with high values of $\mathrm{R}^{2}(0.83$ to 0.97$)$ for all nutrients. Table 4 shows the values that limit each nutrient's sufficiency range calculated by the regression equations.

B

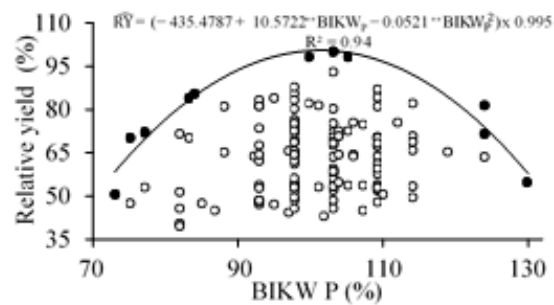

D

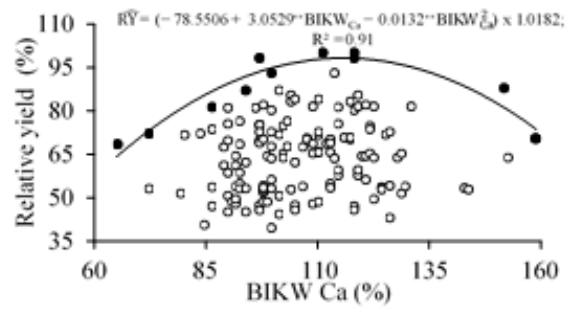

F

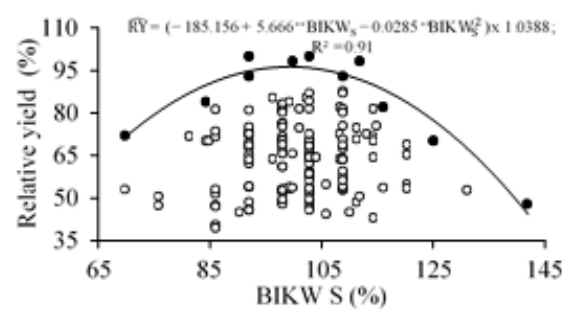

$\mathrm{H}$

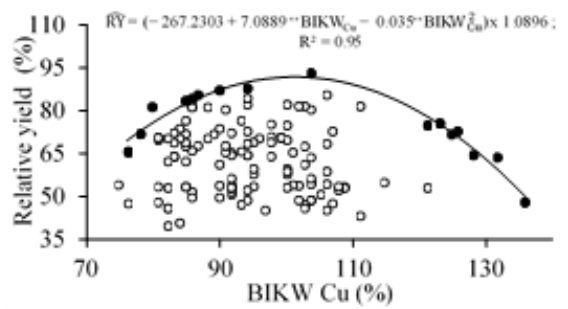

$\mathrm{J}$

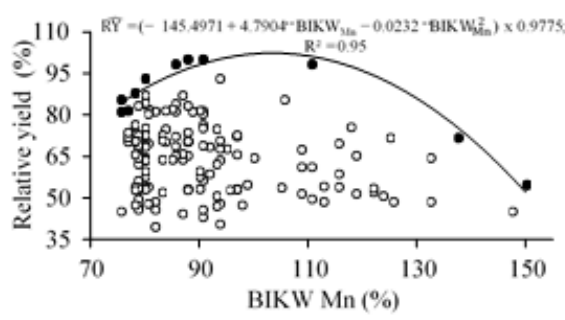

$\mathrm{K}$

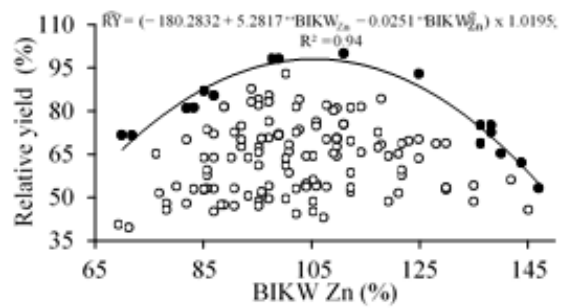

Figure 1. Boundary line fitted to the relationship between relative yield (\%) and Balance Indices of Kenworthy (BIKW) for N (A), P (B), K (C), Ca (D), Mg (E), S (F), B (G), Cu (H), Fe (I), Mn (J) and Zn (K) in 'Grand Nain' banana, Missão Velha-CE, Brazil. **Significant at $\mathrm{p} \leq 0.01$ by $\mathrm{t}$ test; the multipliers 1.095; 0.995; etc. found in the equations correspond to an adjustment factor for the equation to assume the value of $100 \%$ Relative Yield. 

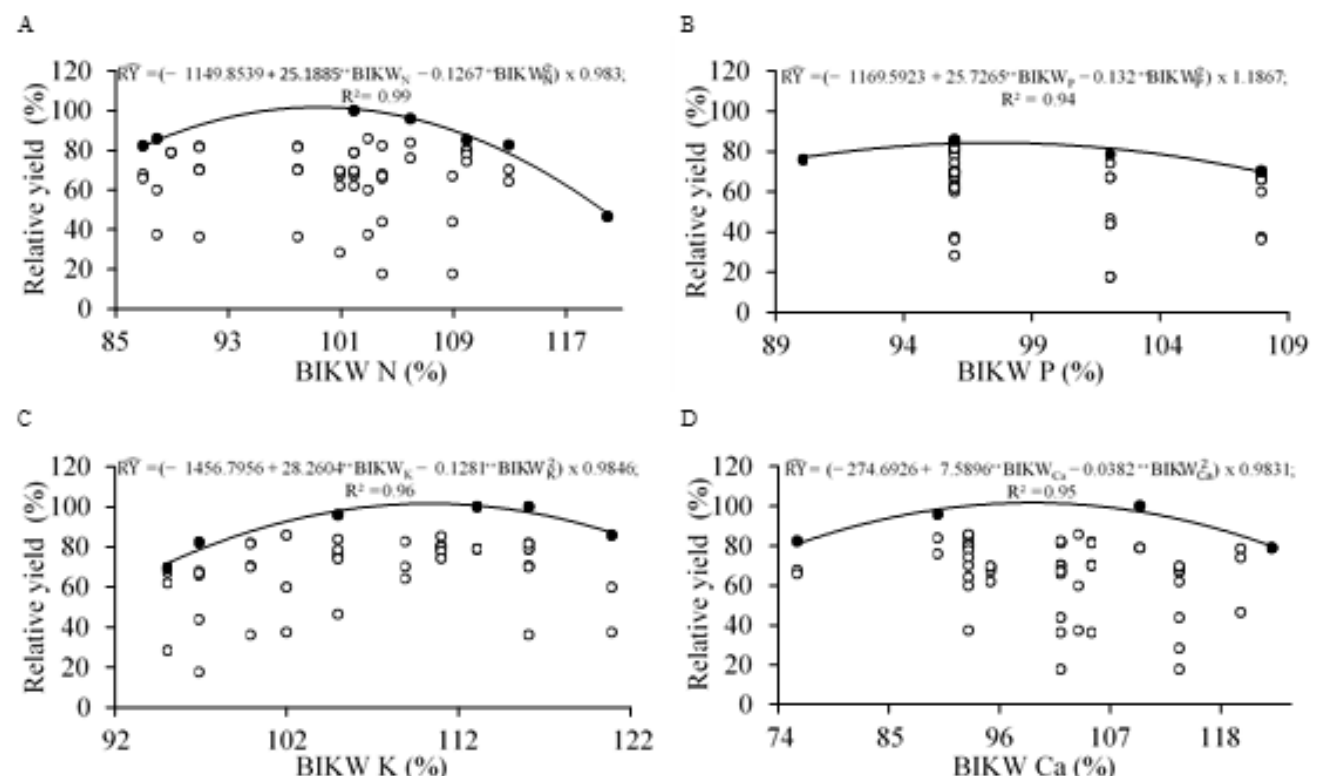

D

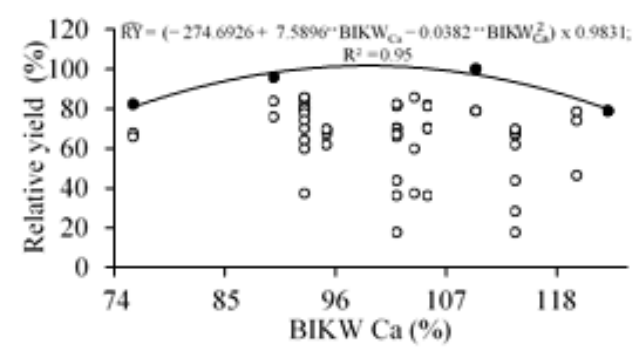

E

F
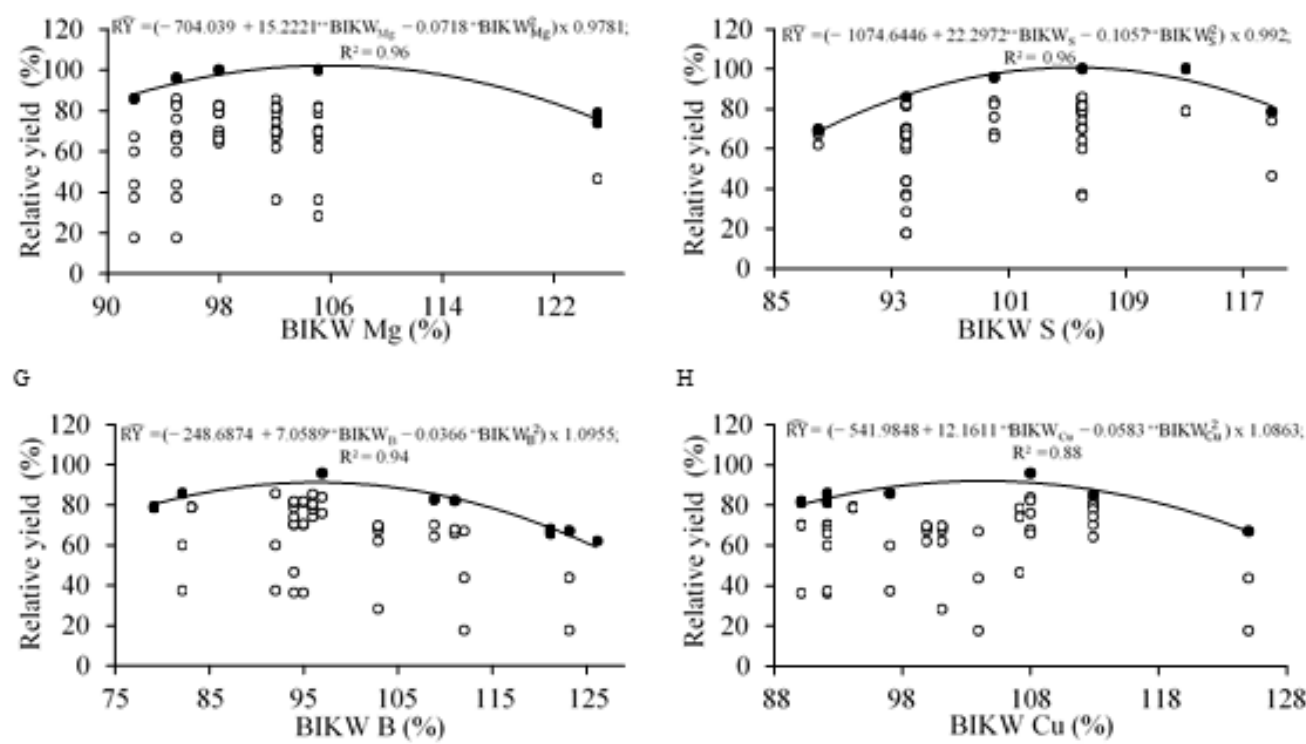

H
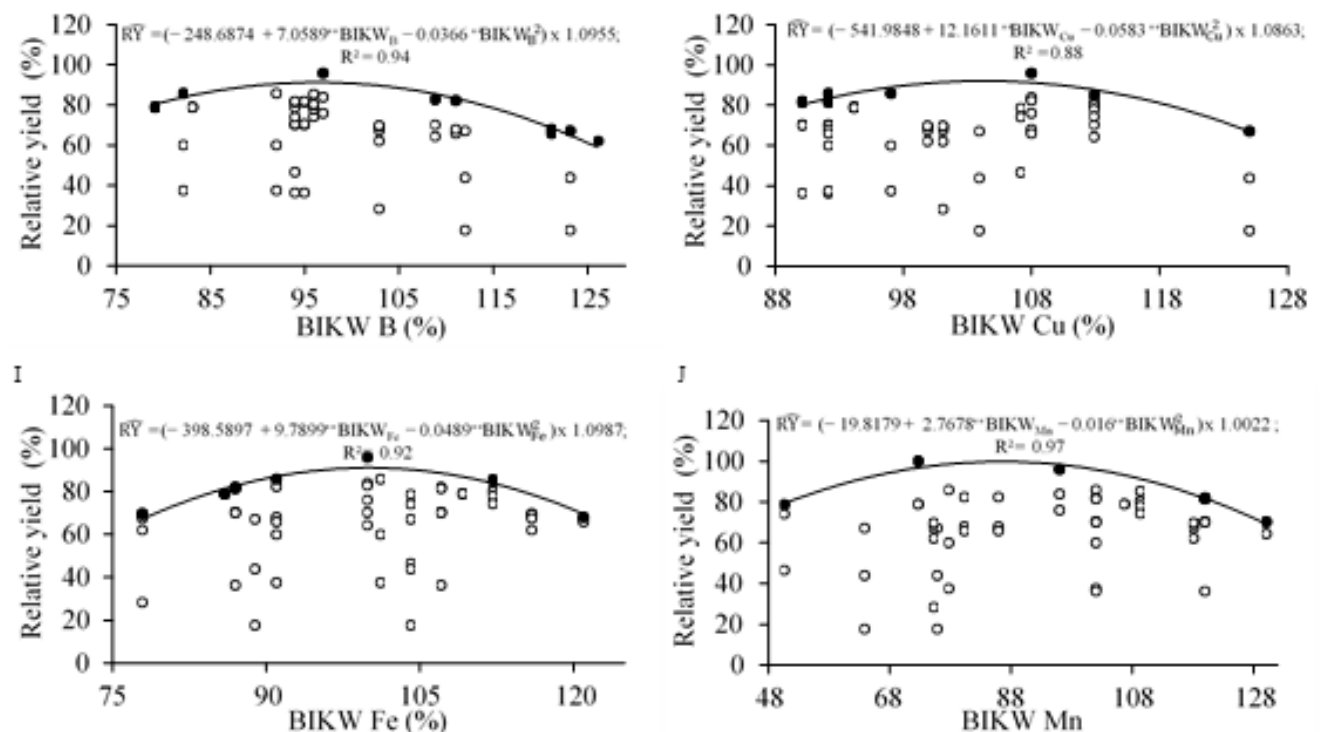

$$
\text { J }
$$

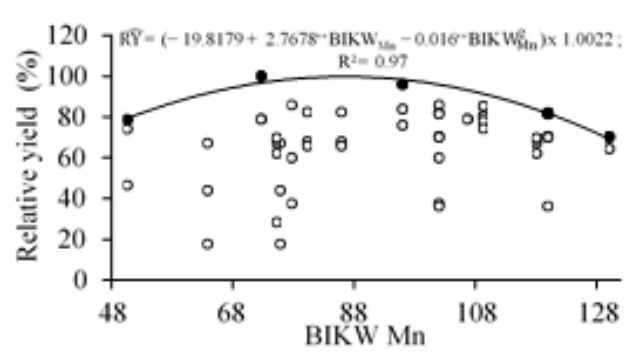

$\mathrm{K}$

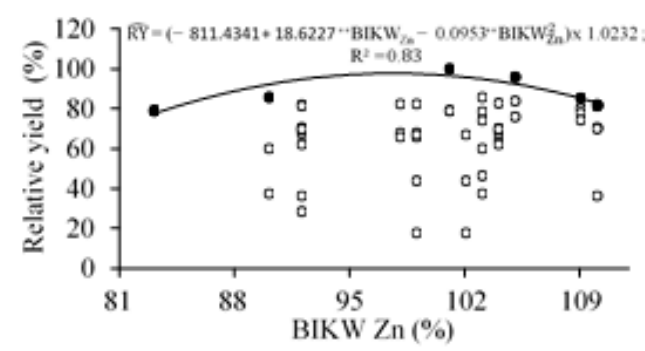

Figure 2. Boundary line fitted to the relationship between relative yield (\%) and Balance Indices of Kenworthy (BIKW) for N (A), P (B), K (C), Ca (D), Mg (E), S (F), B (G), Cu (H), Fe (I), Mn (J) and Zn (K) in 'Grand Nain' banana, Ponto Novo-BA, Brazil. **Significant at $\mathrm{p} \leq 0.01$ by t test; the multipliers $0.983 ; 1.1867$; etc. found in the equations correspond to an adjustment factor for the equation to assume the value of $100 \%$ Relative Yield. 
With the boundary line approach, significant quadratic models with high $\mathrm{R}^{2}(0.78$ to 0.99$)$ were fitted to the relationship between relative yield and leaf nutrient

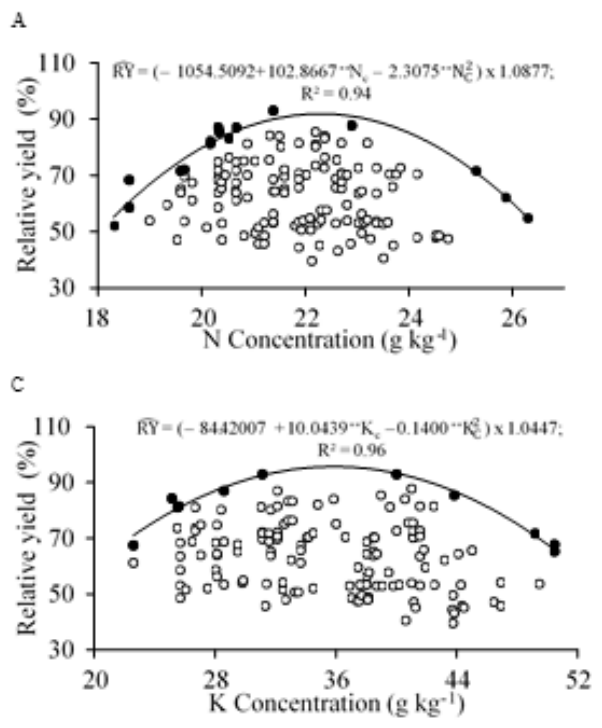

$\mathrm{E}$

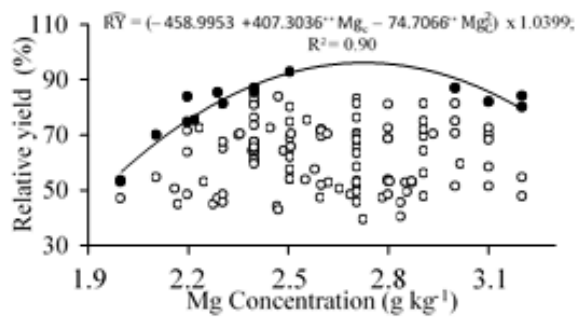

G

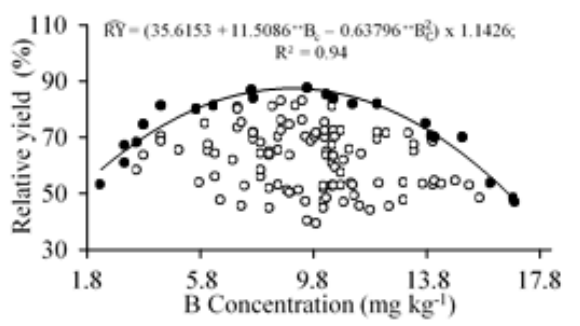
I

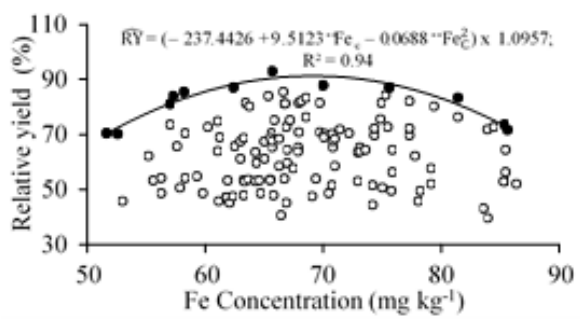

concentrations of 'Grand Nain' banana cultivated in Ceará and Bahia (Figures 3 and 4, respectively). Table 5 shows sufficiency ranges determined based on the concentration ranges proposed in this study.

B

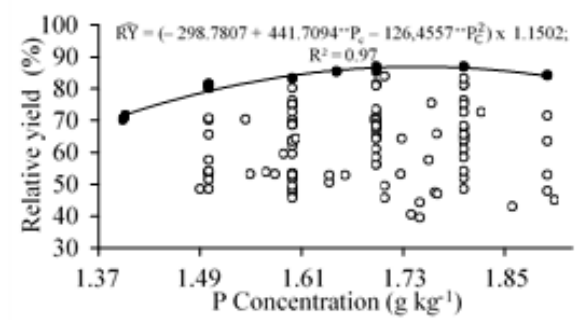

$\mathrm{D}$
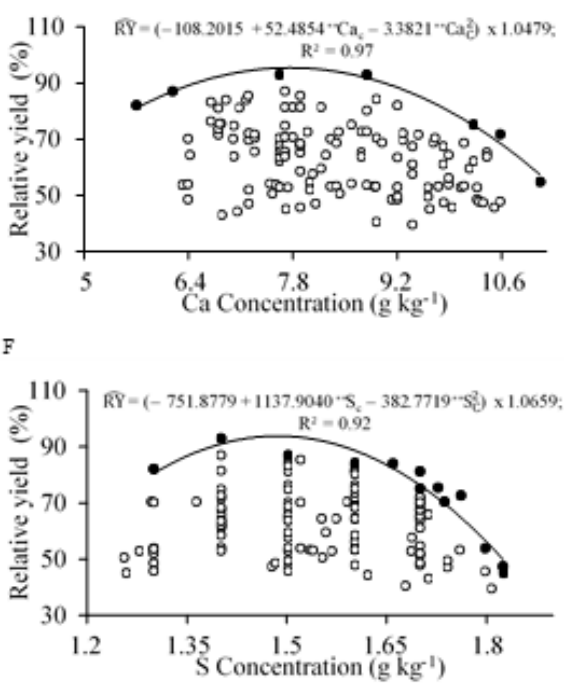

H

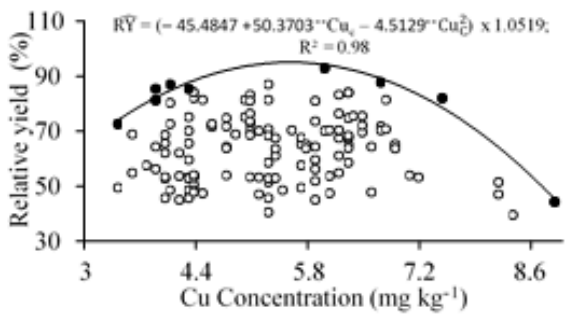

J

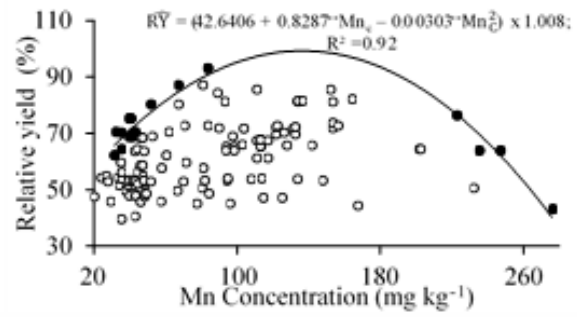

K

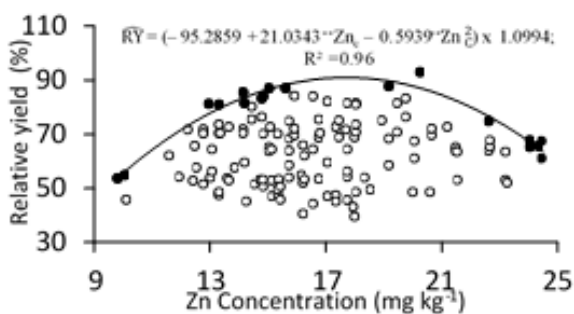

Figure 3. Boundary line fitted to the relationship between relative yield (\%) and leaf concentrations of $N(A), P(B)$, $\mathrm{K}(\mathrm{C}), \mathrm{Ca}(\mathrm{D}), \mathrm{Mg}(\mathrm{E}), \mathrm{S}(\mathrm{F}), \mathrm{B}(\mathrm{G}), \mathrm{Cu}(\mathrm{H}), \mathrm{Fe}(\mathrm{I}), \mathrm{Mn}(\mathrm{J})$ and $\mathrm{Zn}(\mathrm{K})$ in 'Grand Nain' banana, Missão Velha-CE, Brazil. **Significant at $\mathrm{p} \leq 0.01$ by $\mathrm{t}$ test; the multipliers $1.0877 ; 1.1502$; etc. found in the equations correspond to an adjustment factor for the equation to assume the value of $100 \%$ Relative Yield. 
A

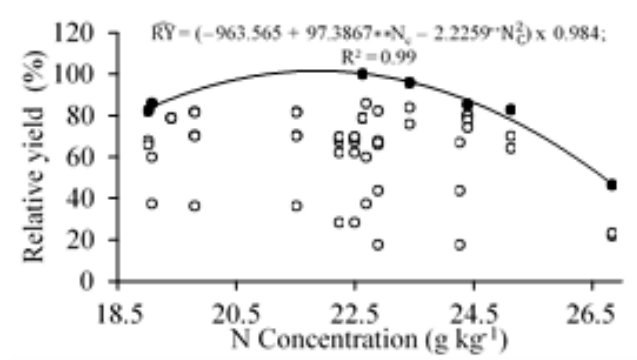

c

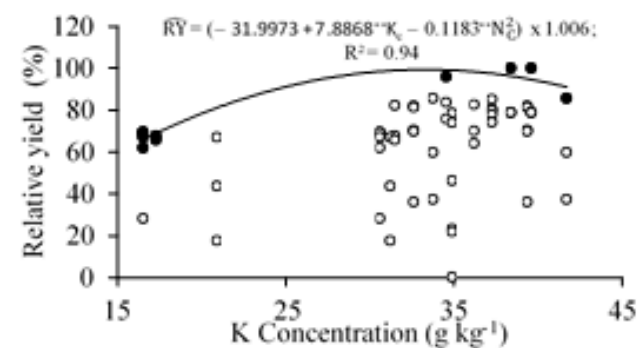

E

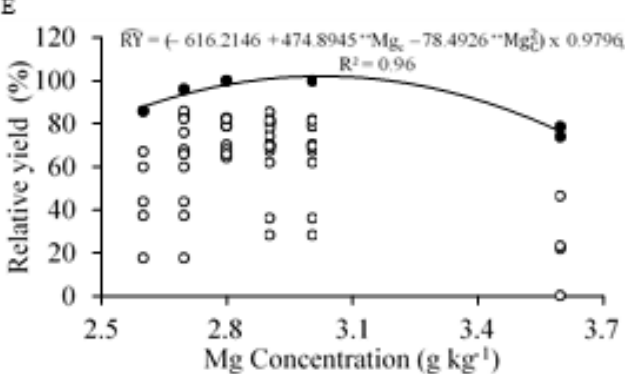

G
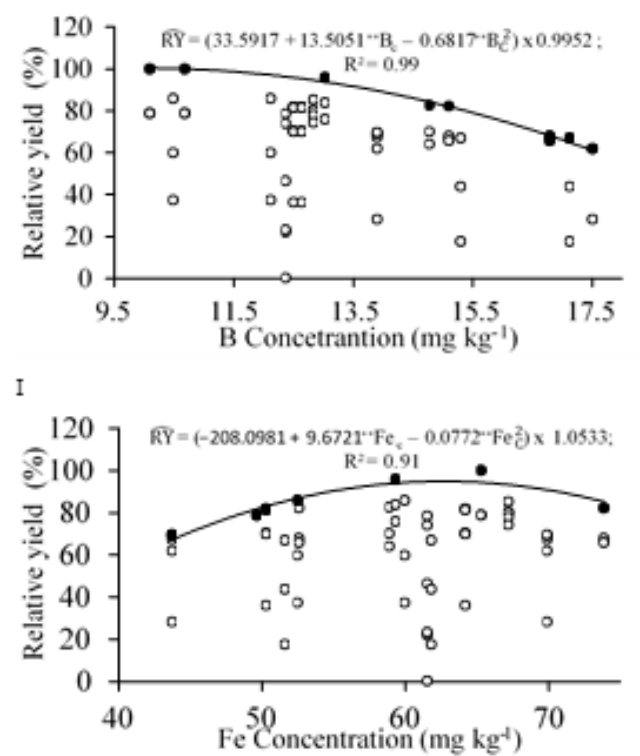

B

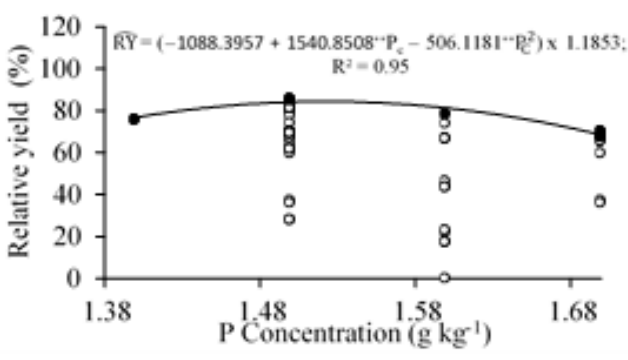

D

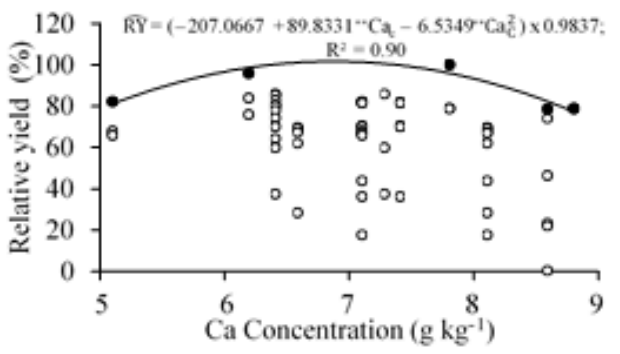

F

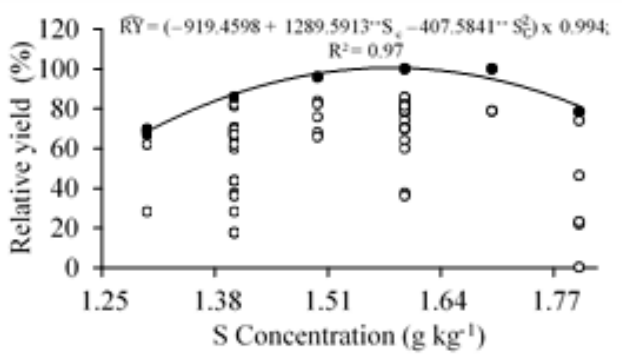

H
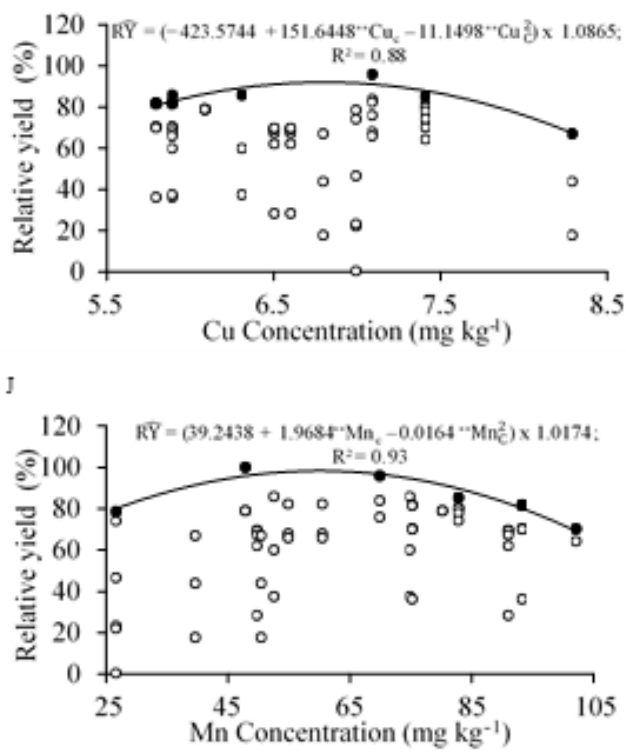

K

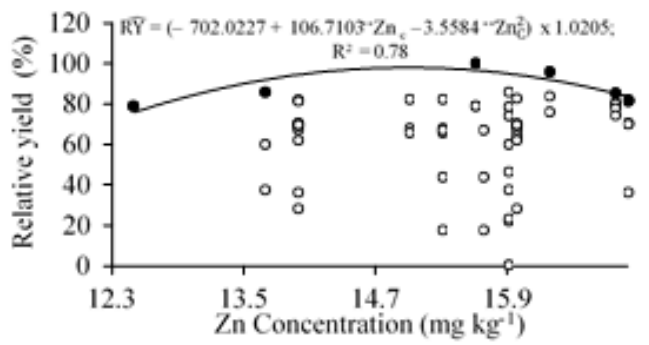

Figure 4. Boundary line fitted to the relationship between relative yield (\%) and leaf concentrations of N (A), P (B), $\mathrm{K}(\mathrm{C}), \mathrm{Ca}(\mathrm{D}), \mathrm{Mg}(\mathrm{E}), \mathrm{S}(\mathrm{F}), \mathrm{B}(\mathrm{G}), \mathrm{Cu}(\mathrm{H}), \mathrm{Fe}(\mathrm{I}), \mathrm{Mn}(\mathrm{J})$ and $\mathrm{Zn}(\mathrm{K})$ in 'Grand Nain' banana, Ponto Novo-BA, Brazil. **Significant at $\mathrm{p} \leq 0.01$ by $\mathrm{t}$ test; the multipliers $0.984 ; 1.1853$; etc. found in the equations correspond to an adjustment factor for the equation to assume the value of $100 \%$ Relative Yield. 
Table 4. Proposed boundary-line concentration ranges for interpreting balance indices of Kenworthy (\%) for 'Grand Nain' bananas grown in Missão Velha-CE, and Ponto Novo-BA, Brazil.

Categories (Reference value - PNRC, Potential Nutrient-Response Curve)

\begin{tabular}{|c|c|c|c|c|c|c|}
\hline Nutrient & $\begin{array}{l}\text { Deficient } \\
(<70 \%)\end{array}$ & $\begin{array}{c}\text { Tendency towards } \\
\text { sufficient } \\
(70-90 \%)\end{array}$ & $\begin{array}{l}\text { Sufficient } \\
(90-100 \%)\end{array}$ & $\begin{array}{c}\text { High } \\
(100-90 \%)\end{array}$ & $\begin{array}{c}\text { Tendency towards } \\
\text { excessive } \\
(90-70 \%)\end{array}$ & $\begin{array}{c}\text { Excessive } \\
(<70 \%)\end{array}$ \\
\hline \multicolumn{7}{|c|}{ Missão Velha - CE } \\
\hline $\mathrm{N}$ & $<87$ & $87-93$ & $93-101$ & $101-110$ & $110-117$ & $\geq 117$ \\
\hline$P$ & $<77$ & $77-88$ & $88-101$ & $101-115$ & $115-125$ & $\geq 125$ \\
\hline K & $<77$ & $77-87$ & $87-101$ & $101-116$ & $116-126$ & $\geq 126$ \\
\hline $\mathrm{Ca}$ & $<69$ & $69-88$ & $88-115$ & $115-143$ & $143-163$ & $\geq 163$ \\
\hline $\mathrm{Mg}$ & $<75$ & $75-88$ & $88-106$ & $106-125$ & $125-138$ & $\geq 138$ \\
\hline S & $<68$ & $68-81$ & $81-99$ & $99-118$ & $118-131$ & $\geq 131$ \\
\hline B & $<62$ & $62-82$ & $82-108$ & $108-135$ & $135-155$ & $\geq 155$ \\
\hline $\mathrm{Cu}$ & $<73$ & $73-85$ & $85-101$ & $101-117$ & $117-129$ & $\geq 129$ \\
\hline $\mathrm{Fe}$ & $<76$ & $76-88$ & $88-104$ & $104-120$ & $120-132$ & $\geq 132$ \\
\hline Mn & $<67$ & $67-82$ & $82-103$ & $103-124$ & $124-140$ & $\geq 140$ \\
\hline $\mathrm{Zn}$ & $<71$ & $71-86$ & $86-105$ & $105-125$ & $125-140$ & $\geq 140$ \\
\hline \multicolumn{7}{|c|}{ Ponto Novo - BA } \\
\hline $\mathrm{N}$ & $<84$ & $84-90$ & $90-99$ & $99-108$ & $108-115$ & $\geq 115$ \\
\hline $\mathrm{P}$ & $<84$ & $84-89$ & $89-97$ & $97-105$ & $105-111$ & $>111$ \\
\hline K & $<82$ & $82-95$ & $95-111$ & $111-128$ & $128-141$ & $\geq 141$ \\
\hline $\mathrm{Ca}$ & $<71$ & $71-83$ & $83-99$ & $99-115$ & $115-127$ & $\geq 127$ \\
\hline $\mathrm{Mg}$ & $<85$ & $85-94$ & $94-106$ & $106-108$ & $108-127$ & $\geq 127$ \\
\hline S & $<89$ & $89-96$ & $96-105$ & $105-115$ & $115-122$ & $\geq 122$ \\
\hline B & $<69$ & $69-81$ & $81-96$ & $96-112$ & $112-124$ & $\geq 124$ \\
\hline $\mathrm{Cu}$ & $<83$ & $83-92$ & $92-104$ & $104-117$ & $117-126$ & $\geq 126$ \\
\hline $\mathrm{Fe}$ & $<76$ & $76-86$ & $86-100$ & $100-114$ & $114-124$ & $\geq 124$ \\
\hline Mn & $<43$ & $43-61$ & $61-86$ & $86-111$ & $111-130$ & $\geq 130$ \\
\hline $\mathrm{Zn}$ & $<80$ & $80-88$ & $88-97$ & $97-108$ & $108-115$ & $\geq 115$ \\
\hline
\end{tabular}


Table 5. Sufficiency ranges determined using the boundary line fitted to the relationship between relative yield and leaf nutrient concentrations for 'Grand Nain' bananas grown in Missão Velha-CE, and Ponto Novo-BA.

Categories (Reference value - PNRC, Potential Nutrient-Response Curve)

\begin{tabular}{|c|c|c|c|c|c|c|}
\hline Nutrient & $\begin{array}{l}\text { Deficient } \\
(<70 \%)\end{array}$ & $\begin{array}{c}\text { Tendency towards } \\
\text { sufficient } \\
(70-90 \%)\end{array}$ & $\begin{array}{l}\text { Sufficient } \\
(90-100 \%)\end{array}$ & $\begin{array}{c}\text { High } \\
(100-90 \%)\end{array}$ & $\begin{array}{c}\text { Tendency towards } \\
\text { excessive } \\
(90-70 \%)\end{array}$ & $\begin{array}{c}\text { Excessive } \\
(<70 \%)\end{array}$ \\
\hline \multicolumn{7}{|c|}{ Missão Velha - CE } \\
\hline \multicolumn{7}{|c|}{ 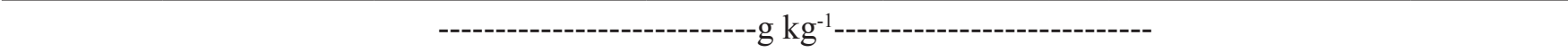 } \\
\hline $\mathrm{N}$ & $<18.6$ & $18.6-20.2$ & $20.2-22.3$ & $22.3-24.5$ & $24.5-26.1$ & $\geq 26.1$ \\
\hline $\mathrm{P}$ & $<1.3$ & $1.3-1.5$ & $1.5-1.7$ & $1.7-1.9$ & $1.9-2.1$ & $\geq 2.1$ \\
\hline K & $<20.1$ & $20.1-27.2$ & $27.2-36.8$ & $36.8-46.5$ & $46.5-53.5$ & $\geq 53.5$ \\
\hline $\mathrm{Ca}$ & $<3.1$ & $3.1-4.8$ & $4.8-6.9$ & $6.9-9.2$ & $9.2-10.8$ & $\geq 10.8$ \\
\hline $\mathrm{Mg}$ & $<1.6$ & $1.6-2.0$ & $2.0-2.6$ & $2.6-3.3$ & $3.3-3.7$ & $\geq 3.7$ \\
\hline S & $<1.1$ & $1.1-1.2$ & $1.2-1.4$ & $1.4-1.6$ & $1.6-1.7$ & $\geq 1.7$ \\
\hline & & thes & ----mg kg-1 & ------------- & & \\
\hline B & $<3.5$ & $3.5-5.9$ & $5.9-9.2$ & $9.2-12.5$ & $12.5-15.0$ & $\geq 15.0$ \\
\hline $\mathrm{Cu}$ & $<1.9$ & $1.9-3.8$ & $3.8-6.5$ & $6.5-9.2$ & $9.2-11.2$ & $\geq 11.2$ \\
\hline $\mathrm{Fe}$ & $<34.5$ & $34.5-49.8$ & $49.8-70.2$ & $70.2-91.5$ & $91.5-106.8$ & $\geq 106.8$ \\
\hline $\mathrm{Mn}$ & $<33.3$ & $33.3-43.2$ & $43.2-79.4$ & $79.4-115.7$ & $115.7-142.2$ & $\geq 142.2$ \\
\hline $\mathrm{Zn}$ & $<10.5$ & $10.5-13.6$ & $13.6-17.8$ & $17.8-22.1$ & $22.1-25.2$ & $\geq 25.2$ \\
\hline \multicolumn{7}{|c|}{ Ponto Novo - BA } \\
\hline \multicolumn{7}{|c|}{-----------------------------g kg-1---------------------------- } \\
\hline $\mathrm{N}$ & $<18.2$ & $18.2-19.7$ & $19.7-21.8$ & $21.8-24.0$ & $24.0-25.6$ & $\geq 25.6$ \\
\hline $\mathrm{P}$ & $<1.3$ & $1.3-1.4$ & $1.4-1.5$ & $1.5-1.7$ & $1.7-1.8$ & $\geq 1.8$ \\
\hline $\mathrm{K}$ & $<17.4$ & $17.4-24.1$ & $24.1-33.2$ & $33.2-42.4$ & $42.4-49.1$ & $\geq 49.1$ \\
\hline $\mathrm{Ca}$ & $<4.7$ & $4.7-5.6$ & $5.6-6.9$ & $6.9-8.1$ & $8.1-9.0$ & $\geq 9.0$ \\
\hline $\mathrm{Mg}$ & $<2.4$ & $2.4-2.7$ & $2.7-3.0$ & $3.0-3.4$ & $3.4-3.7$ & $\geq 3.7$ \\
\hline S & $<1.3$ & $1.3-1.4$ & $1.4-1.6$ & $1.6-1.7$ & $1.7-1.9$ & $\geq 1.9$ \\
\hline \multicolumn{7}{|c|}{----------------------------'mg kg } \\
\hline B & $<3.2$ & $3.2-6.0$ & $6.0-9.8$ & $9.8-13.7$ & $13.7-16.5$ & $\geq 16.5$ \\
\hline $\mathrm{Cu}$ & $<5.2$ & $5.2-5.9$ & $5.9-6.8$ & $6.8-7.7$ & $7.7-8.4$ & $\geq 8.4$ \\
\hline $\mathrm{Fe}$ & $<43.4$ & $43.4-51.5$ & $51.5-62.6$ & $62.6-73.7$ & $73.7-81.8$ & $\geq 81.8$ \\
\hline $\mathrm{Mn}$ & $<17.5$ & $17.5-35.5$ & $35.5-59.9$ & $59.9-84.4$ & $84.4-102.3$ & $\geq 102.3$ \\
\hline $\mathrm{Zn}$ & $<12.1$ & $12.1-13.3$ & $13.3-15.0$ & $15.0-16.6$ & 16.6 - 17.9 & $\geq 17.9$ \\
\hline
\end{tabular}

Based on Kenworthy's norms and concentration ranges determined in this study using the boundary line approach, sufficiency ranges for assessing the nutritional status of 'Grand Nain' bananas cultivated in Ceará and
Bahia were established (Table 6).Using Kenworthy's norms, sufficiency ranges were determined based on interpretive ranges proposed by Kenworthy (1961) (Table $7)$. 
Table 6. Sufficiency ranges determined using boundary-line balance indices of Kenworthy for 'Grand Nain' bananas grown in Missão Velha-CE, and Ponto Novo-BA, Brazil.

\begin{tabular}{|c|c|c|c|c|c|c|}
\hline \multicolumn{7}{|c|}{ Categories (Reference value - PNRC, Potential Nutrient-Response Curve) } \\
\hline Nutrient & $\begin{array}{l}\text { Deficient } \\
(<70 \%)\end{array}$ & $\begin{array}{c}\text { Tendency towards } \\
\text { sufficient } \\
(70-90 \%)\end{array}$ & $\begin{array}{l}\text { Sufficient } \\
(90-100 \%) \\
\end{array}$ & $\begin{array}{c}\text { High } \\
(100-90 \%) \\
\end{array}$ & $\begin{array}{c}\text { Tendency towards } \\
\text { excessive } \\
(90-70 \%)\end{array}$ & $\begin{array}{c}\text { Excessive } \\
(<70 \%)\end{array}$ \\
\hline \multicolumn{7}{|c|}{ Missão Velha - CE } \\
\hline \multicolumn{7}{|c|}{ 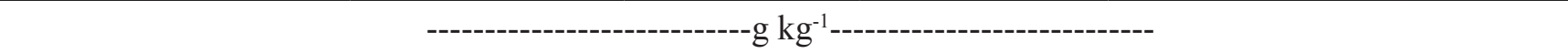 } \\
\hline $\mathrm{N}$ & $<18.7$ & $18.7-20.1$ & $20.1-22.0$ & $22.0-24.1$ & $24.1-25.8$ & $\geq 25.8$ \\
\hline $\mathrm{P}$ & $<1.2$ & $1.2-1.4$ & $1.4-1.7$ & $1.7-1.9$ & $1.9-2.1$ & $\geq 2.1$ \\
\hline $\mathrm{K}$ & $<26.1$ & $26.1-30.5$ & $30.5-36.6$ & $36.6-43.2$ & $43.2-47.6$ & $\geq 47.6$ \\
\hline $\mathrm{Ca}$ & $<4.7$ & $4.7-6.6$ & $6.6-9.4$ & $9.4-12.3$ & $12.3-14.4$ & $\geq 14.4$ \\
\hline $\mathrm{Mg}$ & $<1.8$ & $1.8-2.2$ & $2.2-2.8$ & $2.8-3.4$ & $3.4-3.8$ & $\geq 3.8$ \\
\hline $\mathrm{S}$ & $<1.0$ & $1.0-1.2$ & $1.2-1.5$ & $1.5-1.9$ & $1.9-2.1$ & $\geq 2.1$ \\
\hline & & & -----mg kg & '--------- & & \\
\hline $\mathrm{B}$ & $<4.3$ & $4.3-7.1$ & $7.1-10.8$ & $10.8-14.6$ & $14.6-17.4$ & $\geq 17.4$ \\
\hline $\mathrm{Cu}$ & $<3.0$ & $3.0-4.3$ & $4.3-6.0$ & $6.0-7.7$ & $7.7-9.0$ & $\geq 9.0$ \\
\hline $\mathrm{Fe}$ & $<49.1$ & $49.1-58.9$ & $58.9-72.0$ & $72.0-85.0$ & $85.0-94.8$ & $\geq 94.8$ \\
\hline $\mathrm{Mn}$ & & $59.6^{1}$ & $59.6-187.1$ & $187.1-314.5$ & $314.5-411.6$ & $\geq 411.6$ \\
\hline $\mathrm{Zn}$ & $<10.2$ & $10.2-13.1$ & $13.1-16.8$ & $16.8-20.6$ & $20.6-23.5$ & $\geq 23.5$ \\
\hline \multicolumn{7}{|c|}{ Ponto Novo - BA } \\
\hline \multicolumn{7}{|c|}{ 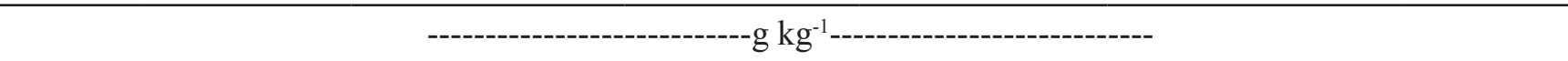 } \\
\hline $\mathrm{N}$ & $<18.2$ & $18.2-19.6$ & $19.6-21.8$ & $21.8-23.9$ & $23.9-25.6$ & $\geq 25.6$ \\
\hline $\mathrm{P}$ & $<1.3$ & $1.3-1.4$ & $1.4-1.5$ & $1.5-1.6$ & $1.6-1.7$ & $\geq 1.7$ \\
\hline $\mathrm{K}$ & $<24.9$ & $24.9-30.5$ & $30.5-37.3$ & $37.3-44.6$ & $44.6-50.2$ & $\geq 50.2$ \\
\hline $\mathrm{Ca}$ & $<4.7$ & $4.7-5.6$ & $5.6-6.9$ & $6.9-8.2$ & $8.2-9.1$ & $\geq 9.1$ \\
\hline $\mathrm{Mg}$ & $<2.4$ & $2.4-2.7$ & $2.7-3.0$ & $3.0-3.1$ & $3.1-3.7$ & $\geq 3.7$ \\
\hline $\mathrm{S}$ & $<1.3$ & $1.3-1.4$ & $1.4-1.6$ & $1.6-1.7$ & $1.7-1.9$ & $\geq 1.9$ \\
\hline \multicolumn{7}{|c|}{ 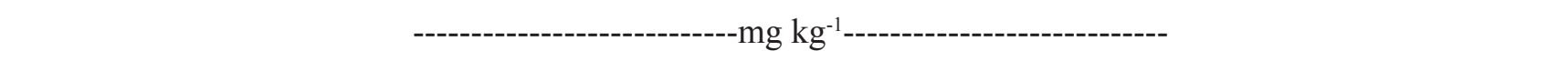 } \\
\hline $\mathrm{B}$ & $<8.5$ & $8.5-10.4$ & $10.4-12.8$ & $12.8-15.3$ & $15.3-17.2$ & $\geq 17.2$ \\
\hline $\mathrm{Cu}$ & $<5.3$ & $5.3-5.9$ & $5.9-6.8$ & $6.8-7.7$ & $7.7-8.4$ & $\geq 8.4$ \\
\hline $\mathrm{Fe}$ & $<42.3$ & $42.3-49.3$ & $49.3-59.0$ & $59.0-68.8$ & $68.8-75.8$ & $\geq 75.8$ \\
\hline $\mathrm{Mn}$ & $<19.1$ & $19.1-36.3$ & $36.3-60.2$ & $60.2-84.0$ & $84.0-102.2$ & $\geq 102.2$ \\
\hline $\mathrm{Zn}$ & $<12.0$ & $12.0-13.4$ & $13.4-14.9$ & $14.9-16.7$ & $16.7-17.9$ & $\geq 17.9$ \\
\hline
\end{tabular}


Table 7. Leaf nutrient sufficiency ranges based on limits calculated by the original method of Kenworthy (1961) for 'Grand Nain' bananas cultivated in Missão Velha-CE, and Ponto Novo-BA, Brazil.

\begin{tabular}{|c|c|c|c|c|c|c|}
\hline \multicolumn{7}{|c|}{ Categories (Reference Value - Balance Index of Kenworthy) } \\
\hline Nutrient & Deficient & $\begin{array}{c}\text { Tendency towards } \\
\text { sufficient }\end{array}$ & Sufficient & High & $\begin{array}{c}\text { Tendency towards } \\
\text { excessive }\end{array}$ & Excessive \\
\hline & $(<50 \%)$ & $(50-83 \%)$ & $(83-100 \%)$ & $(100-117 \%)$ & $(117-150 \%)$ & $(\geq 150 \%)$ \\
\hline \multicolumn{7}{|c|}{ Missão Velha - CE } \\
\hline \multicolumn{7}{|c|}{-----------------------------g kg-1'--------------------------- } \\
\hline $\mathrm{N}$ & $<9.9$ & $9.9-17.7$ & $17.7-21.8$ & $21.8-25.8$ & $25.8-33.7$ & $\geq 33.7$ \\
\hline $\mathrm{P}$ & $<0.7$ & $0.7-1.3$ & $1.3-1.6$ & $1.6-2.0$ & $2.0-2.6$ & $\geq 2.6$ \\
\hline $\mathrm{K}$ & $<14.3$ & $14.3-28.7$ & $28.7-36.2$ & $36.2-43.7$ & $43.7-58.1$ & $\geq 58.1$ \\
\hline $\mathrm{Ca}$ & $<2.7$ & $2.7-6.1$ & $6.1-7.9$ & $7.9-9.7$ & $9.7-13.1$ & $\geq 13.1$ \\
\hline $\mathrm{Mg}$ & $<1.1$ & $1.1-2.1$ & $2.1-2.6$ & $2.6-3.1$ & $3.1-4.1$ & $\geq 4.1$ \\
\hline $\mathrm{S}$ & $<0.6$ & $0.6-1.2$ & $1.2-1.5$ & $1.5-1.9$ & $1.9-2.5$ & $\geq 2.5$ \\
\hline & & & --mg kg & 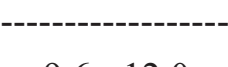 & & \\
\hline $\mathrm{B}$ & $<2.6$ & $2.6-7.2$ & $7.2-9.6$ & $9.6-12.0$ & $12.0-16.7$ & $\geq 16.7$ \\
\hline $\mathrm{Cu}$ & $<0.6$ & $0.6-4.1$ & $4.1-5.9$ & $5.9-7.7$ & $7.7-11.2$ & $\geq 11.2$ \\
\hline $\mathrm{Fe}$ & $<27.9$ & $27.9-54.8$ & $54.8-68.7$ & $68.7-82.6$ & $82.6-109.5$ & $\geq 109.5$ \\
\hline $\mathrm{Mn}$ & & $65.7^{1}$ & $65.7-168.9$ & $168.9-272.0$ & $272.0-472.3$ & $\geq 472.3$ \\
\hline $\mathrm{Zn}$ & $<6.1$ & $6.1-12.5$ & $12.5-15.8$ & $15.8-19.1$ & $19.1-25.5$ & $\geq 25.5$ \\
\hline \multicolumn{7}{|c|}{ Ponto Novo - BA } \\
\hline \multicolumn{7}{|c|}{ 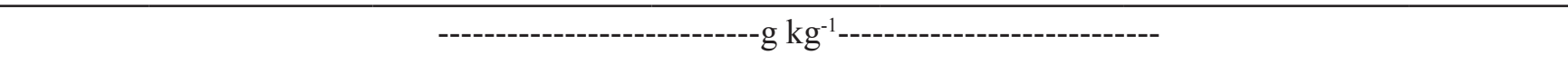 } \\
\hline $\mathrm{N}$ & $<10.0$ & $10.0-17.9$ & $17.9-22.0$ & $22.0-26.1$ & $26.1-34.0$ & $\geq 34.0$ \\
\hline $\mathrm{P}$ & $<0.7$ & $0.7-1.3$ & $1.3-1.6$ & $1.6-1.9$ & $1.9-2.4$ & $\geq 2.4$ \\
\hline $\mathrm{K}$ & $<11.2$ & $11.2-25.4$ & $25.4-32.6$ & $32.6-39.9$ & $39.9-54.0$ & $\geq 54.0$ \\
\hline $\mathrm{Ca}$ & $<3.0$ & $3.0-5.6$ & $5.6-7.0$ & $7.0-8.3$ & $8.3-11.0$ & $\geq 11.0$ \\
\hline $\mathrm{Mg}$ & $<1.4$ & $1.4-2.4$ & $2.4-2.9$ & $2.9-3.4$ & $3.4-4.4$ & $\geq 4.4$ \\
\hline $\mathrm{S}$ & $<0.7$ & $0.7-1.2$ & $1.2-1.5$ & $1.5-1.8$ & $1.8-2.3$ & $\geq 2.3$ \\
\hline & & & ----mg kg-1 & - & --- & \\
\hline $\mathrm{B}$ & $<5.4$ & $5.4-10.7$ & $10.7-13.4$ & $13.4-16.1$ & $16.1-21.4$ & $\geq 21.4$ \\
\hline $\mathrm{Cu}$ & $<2.9$ & $2.9-5.3$ & $5.3-6.5$ & $6.5-7.7$ & $7.7-10.1$ & $\geq 10.1$ \\
\hline $\mathrm{Fe}$ & $<24.1$ & $24.1-47.2$ & $47.2-59.0$ & $59.0-70.9$ & $70.9-93.9$ & $\geq 93.9$ \\
\hline $\mathrm{Mn}$ & $<25.8$ & $25.8-57.3$ & $57.3-73.5$ & $73.5-89.8$ & $89.8-121.3$ & $\geq 121.3$ \\
\hline $\mathrm{Zn}$ & $<7.0$ & $7.0-12.5$ & $12.5-15.4$ & $15.4-18.3$ & $18.3-23.8$ & $\geq 23.8$ \\
\hline
\end{tabular}

Regardless of the nutrient, interpretive concentration ranges established using the original method of Kenworthy (1961) do not differ from one another because this method is based on the assumption that each nutrient limits the crop yield to the same extent; nonetheless, quadratic models (Figure 1 and 2) fitted to the relationship between relative yield and balance indices of Kenworthy determined sufficiency ranges with different limits for each nutrient, which contradicts the Kenworthy's approach. Differences across sufficiency ranges are due to how much a nutrient limits the yield. This yield-limiting effect is linked to how limiting the nutrient is when its concentration is lowest.
This indicates that interpreting indices of Kenworthy while overlooking the differences in sufficiency ranges inherent to the crop or nutrient is not the best approach. Apparently, sufficiency ranges originally proposed by Kenworthy for apple trees had only followed a statistical criterion that took into account a $20 \%$ variability within mean leaf nutrient levels from a reference population; as a result, the farther the variability from $20 \%$, the more misleading interpretations. Misleading interpretations are more likely to occur (DONATO et al., 2010) due to the complexity of micronutrient dynamics in the soil-plant system (ABREU et al., 2007). 
With the exception of $\mathrm{N}$, lower limits of sufficient concentration ranges for 'Grand Nain' banana cultivated in Ceará (Table 4) were close to $83 \%$, the value proposed by Kenworthy (1961). Upper limits were also consistently close to the upper limit of $100 \%$ determined by the original method of Kenworthy, with the exception of $\mathrm{Ca}(115 \%)$. Lower limits of sufficient ranges for 'Grand Nain' grown in Bahia differed from that reported by Kenworthy (1961) for most nutrients including N (90\%), K (95\%), S (96\%), $\mathrm{Mg}(94 \%), \mathrm{Cu}(92 \%)$ and $\mathrm{Mn}(61 \%)$. The upper limits, however, of most nutrients were close to $100 \%$, with the exception of K (111\%) and Mn (86\%).

Sufficient nitrogen concentrations established using the boundary line approach for 'Grand Nain' cultivated in Ceará ranged from 20.2 to $22.3 \mathrm{~g} \mathrm{~kg}^{-1}$ (Table 5), whose limits are similar to the modified method of Kenworthy, 20.1 to $22.0 \mathrm{~g} \mathrm{~kg}^{-1}$ (Table 6), but different from the original method of Kenworthy, 17.9 to $21.8 \mathrm{~g} \mathrm{~kg}^{-1}$ (Table 7). In Bahia, the sufficient N range for 'Grand Nain' was 19.7$21.8 \mathrm{~g} \mathrm{~kg}^{-1}$ (Table 5), similar to values determined by the modified method of Kenworthy, 19.6-21.8 $\mathrm{g} \mathrm{kg}^{-1}$ (Table 6), but different from the original method, 17.9-22.0 $\mathrm{g}$ $\mathrm{kg}^{-1}$ (Table 7).

Nitrogen sufficiency ranges proposed herein differ from that (27.0-36.0 $\left.\mathrm{g} \mathrm{kg}^{-1}\right)$ proposed by Quaggio and Raij (1997) and is close to the lower limit of the range (17.036. $\mathrm{g} \mathrm{kg}^{-1}$ ) proposed by Pauletti and Motta (2017). The authors proposed the range for bananas of the Cavendish subgroup, to which 'Grand Nain' also belongs, cultivated in the Brazilian state of São Paulo and Paraná, respectively.

Investigations carried out in Brazil showed that bananas grown on sandy soils with low organic matter content respond well to $\mathrm{N}$ fertilizer application, especially in the first cycle (SILVA et al., 2012). However, when grown on soil with medium-to-high organic matter content $\left(>16 \mathrm{~g} \mathrm{~kg}^{-1}\right)$, bananas might not respond to nitrogen fertilization. Yields may even decrease as $\mathrm{N}$ becomes excessive due to increased plant residues mineralization rate and organic matter content. This is further enhanced by high temperature and high soil moisture due to irrigation (SILVA et al., 2012; SILVA et al., 2013; PULITO et al., 2015).

Crop residue returning to the soil after harvest is an important source of nutrients in banana plantations since about $100 \mathrm{~kg} \mathrm{ha}^{-1}$ year ${ }^{-1}$ of nitrogen return to the soil through biogeochemical cycling (HOFFMANN et al., 2010). Higher $\mathrm{N}$ availability in soils with mediumto-high organic matter content, and the consequent reduction in crop yield possibly due to excessive N, may have contributed to the lower sufficient $\mathrm{N}$ concentration range in comparison with the range reported by Quaggio and Raij (1997).
Sufficient phosphorus concentration range established using the boundary line approach for 'Grand Nain' grown in Ceará was 1.5-1.7 $\mathrm{g} \mathrm{kg}^{-1}$ (Table 5), which are similar to those determined by both modified and original methods of Kenworthy, 1.4-1.7 $\mathrm{g} \mathrm{kg}^{-1}$ (Table 6) and 1.3-1.6 $\mathrm{g} \mathrm{kg}^{-1}$ (Table 7), respectively. In Bahia, the sufficient range derived from the boundary line for 'Grand Nain' ranged from 1.4 to $1.5 \mathrm{~g} \mathrm{~kg}^{-1}$ (Table 5), whose limits are equal to limits established by the modified method of Kenworthy (Table 6) and similar to the original method of Kenworthy, 1.3-1.6 $\mathrm{g} \mathrm{kg}^{-1}$ (Table 7). Upper limits reported herein, both in Ceará and Bahia, are consistent with lower limits established by Quaggio and Raij (1997), 1.8-2.7 g $\mathrm{kg}^{-1}$, and Pauletti and Motta (2017), 1.6-3.2 $\mathrm{g} \mathrm{kg}^{-1}$.

Phosphorus is the least accumulated macronutrient in 'Grand Nain' banana (HOFFMANN et al., 2010). Despite higher $\mathrm{P}$ availability (Table 1 ), there was no increase in P leaf concentration as reported by Quaggio and Raij (1997). Higher P concentration might reduce yield due to imbalances with other nutrients, particularly Zn. Multivariate compositional nutrient diagnosis (CND) indices developed by Raghupathi et al. (2002) were instrumental in diagnosing $\mathrm{P}$ and $\mathrm{Zn}$ imbalances caused by changes in N and $\mathrm{K}$ concentrations in Robusta (AAA) and Ney Poovan $(\mathrm{AB})$ cultivars of banana. Although $\mathrm{P}$ is more available in the soil, $P$ leaf concentration remains virtually unchanged (DAMATTO JUNIOR et al., 2011), even among cultivars of different subgroups (BORGES et al., 2006).

Sufficient potassium concentrations derived from boundary line for 'Grand Nain' grown in Ceará ranged from 27.2 to $36.8 \mathrm{~g} \mathrm{~kg}^{-1}$ (Table 5), which is wider than those established by modified 30.5-36.6 $\mathrm{g} \mathrm{kg}^{-1}$ (Table 6), and original method of Kenworthy, 28.7 to $36.2 \mathrm{~g} \mathrm{~kg}^{-1}$ (Table 7). Sufficient K concentrations determined using the boundary line approach for 'Grand Nain' in Bahia ranged from 24.1 to $33.2 \mathrm{~g} \mathrm{~kg}^{-1}$ (Table 5). This range is different from the one calculated with the modified method of Kenworthy, 30.5-37.3 $\mathrm{g} \mathrm{kg}^{-1}$ (Table 6) and similar to the original method of Kenworthy, 25.4-32.6 g kg-1 (Table 7). Upper limits of these ranges are close to lower limits reported by Quaggio and Raij (1997), 35.0-54.0 $\mathrm{g} \mathrm{kg}^{-1}$, and Pauletti and Motta (2017), 24.0-56.0 $\mathrm{g} \mathrm{kg}^{-1}$.

Potassium is the most absorbed nutrient by bananas and hence demanded in large amounts (HOFFMANN et al., 2010). Higher K availability in the soil in association with high $\mathrm{K}_{2} \mathrm{O}$ fertilization rates leads to higher leaf $\mathrm{K}$ concentration; however, increased leaf $\mathrm{K}$ concentration does not necessarily increase crop yield, which may be either luxury or excessive consumption; instead, crop yield might reduce due to excessive $\mathrm{K}$ and/or to imbalances with other nutrients, especially Mg. Balanced $\mathrm{K}$ fertilization reflects in leaf $\mathrm{K}$ concentrations as well as $\mathrm{Ca}$ and $\mathrm{Mg}$ concentrations, with which $\mathrm{K}$ greatly interacts (SILVA; CARVALHO, 2004). 
The lower sufficient range proposed by this study is consistent with the decrease in $\mathrm{K}_{2} \mathrm{O}$ rates that have been recommended over the years. For example, the recommendation in 1999 for northern Minas Gerais $\mathrm{K}_{2} \mathrm{O}$ rates that ranged from 800 to $1,600 \mathrm{~kg} \mathrm{ha}^{-1}$ year ${ }^{-1}$ decreased to ranges of 675 to $1,050 \mathrm{~kg} \mathrm{ha}^{-1} \mathrm{year}^{-1}$ (SILVA, 2015).

Decreased $\mathrm{K}_{2} \mathrm{O}$ fertilizer rates and the consequent decreased $\mathrm{K}$ sufficient range in bananas are supported by: biochemical cycling, inside the plant, from older to younger tissues; from plant pseudostems, left standing after the harvest, to suckers linked to it; and/ or biogeochemically, when the pseudostem is cut down and left on the soil to decompose right after harvesting. Potassium cycling is particularly important from the second cycle on by providing the soil with 70\% (SOTO BALLESTERO, 2008) to $80 \%$ (HOFFMANN et al., 2010) of the potassium taken up by 'Grand Nain' bananas.

Sufficient calcium concentrations established using the boundary line approach for 'Grand Nain' cultivated in Ceará ranged from 4.8 to $6.9 \mathrm{~g} \mathrm{~kg}^{-1}$ (Table 5), different from the modified and original method of Kenworthy, 6.6-9.4 $\mathrm{g} \mathrm{kg}^{-1}$ (Table 6) and 6.1-7.9 $\mathrm{g} \mathrm{kg}^{-1}$ (Table 7), respectively. In Bahia, the same sufficient range (5.6-6.9 $\mathrm{g} \mathrm{kg}^{-1}$ ) was established using the boundary line approach (Table 5) and the modified method of Kenworthy (Table 6). The original method of Kenworthy determined a slightly different range, 5.6-7.0 $\mathrm{g} \mathrm{kg}^{-1}$ (Table 7). The ranges, regardless of the site, were narrower than Ca sufficient range established by Quaggio and Raij (1997), 3.0-12.0 g $\mathrm{kg}^{-1}$, and Pauletti and Motta (2017), 5.0-12.0 $\mathrm{g} \mathrm{kg}^{-1}$.

Sufficient magnesium concentrations established using the boundary line approach for 'Grand Nain' cultivated in Ceará ranged from 2.0 to $2.6 \mathrm{~g} \mathrm{~kg}^{-1}$ (Table 5 ); similar ranges were established by the modified and original methods of Kenworthy, 2.2-2.8 g kg${ }^{-1}$ (Table 6) and 2.1-2.6 $\mathrm{g} \mathrm{kg}^{-1}$ (Table 7), respectively. Equal sufficient ranges were established using the boundary line approach and modified method of Kenworthy for 'Grand Nain' grown in Bahia, 2.7-3.0 $\mathrm{g} \mathrm{kg}^{-1}$ (Table 5 and 6). The original method of Kenworthy generated a similar range, 2.4-2.9 $\mathrm{g} \mathrm{kg}^{-1}$ (Table 7). These ranges differ from that proposed by Quaggio and Raij (1997), 3.0-6.0 $\mathrm{g} \mathrm{kg}^{-1}$, whose lower limit is similar to the upper limits of the ranges reported herein, regardless of the site, however, the lower limit is close to the range proposed by Pauletti and Motta (2017), 2.2-4.0 $\mathrm{g} \mathrm{kg}^{-1}$.

The lower Mg concentration ranges might be due to higher $\mathrm{K}$ fertilizer rates, decreasing $\mathrm{Mg}$ uptake from the soil (SILVA; CARVALHO, 2004). One must be careful when diagnosing the nutritional status of bananas; the crop is a monocot and has low root cation exchange capacity, thus absorbing monovalent rather than divalent ions. This can accentuate $\mathrm{Mg}$ and $\mathrm{Ca}$ deficiencies in bananas. A balanced supply of $\mathrm{K}, \mathrm{Ca}$ and $\mathrm{Mg}$ is essential in alleviating plant stress (MARSCHNER, 2012), which is particularly important for crops grown in the semiarid.
Sufficient sulfur concentrations generated using the boundary line approach for 'Grand Nain' grown in Ceará ranged from 1.2 to $1.4 \mathrm{~g} \mathrm{~kg}^{-1}$ (Table 5). Similar limits were established by both modified and original methods of Kenworthy: 1.2-1.5 $\mathrm{g} \mathrm{kg}^{-1}$ (Table 6 and 7). For 'Grand Nain' grown in Bahia, sufficient ranges generated using the boundary line approach and modified method of Kenworthy had the same limits, $1.4-1.6 \mathrm{~g} \mathrm{~kg}^{-1}$ (Table 5 and 6). The range established by the original method was different, 1.2-1.5 $\mathrm{g} \mathrm{kg}^{-1}$ (Table 7); nonetheless, every $\mathrm{S}$ range differs from the range reported by Quaggio and Raij (1997), 1.6-3.0 $\mathrm{g} \mathrm{kg}^{-1}$, and by Pauletti and Motta (2017), $1.5-2.4 \mathrm{~g} \mathrm{~kg}^{-1}$.

Although macronutrient concentration ranges were different from one another in cultivars of the same subgroup, Raghupathi et al. (2002) compared, through discriminant analysis, two quite distinct banana cultivars, Robusta (AAA) and Ney Poovan (AB), and reported differences in nutrient concentration range only for $\mathrm{K}$ and $\mathrm{Ca}$. The authors used multivariate compositional nutrient diagnosis (CND), which shows that the method plays a role in determining the extent to which the sufficiency ranges differ from one another.

Sufficient boron concentrations derived from boundary line ranged from 5.9 to $9.2 \mathrm{mg} \mathrm{kg}^{-1}$ (Table 5); somewhat similar to ranges established by the modified and original methods of Kenworthy: 7.1-10.8 mg kg ${ }^{1}$ (Table 6) and 7.2-9.6 $\mathrm{mg} \mathrm{kg}^{-1}$ (Table 7), respectively. In Bahia, the sufficient concentrations estimated from boundary line ranged from 6.0 to $9.8 \mathrm{mg} \mathrm{kg}^{-1}$ (Table 5); different from ranges established by the modified and original methods of Kenworthy: 10.4-12.8 mg kg${ }^{-1}$ (Table 6) and 10.7-13.4 $\mathrm{mg} \mathrm{kg}^{-1}$ (Table 7), respectively. Upper concentration limits of ranges derived from boundary line for 'Grand Nain' grown in Ceará are close to the lower limit established by Quaggio and Raij (1997): 10.0-25.0 $\mathrm{mg} \mathrm{kg}^{-1}$, while its lower limit is similar to those established by the modified and original method of Kenworthy in Bahia. A broader range, but with lower limits similar to that of the present work is reported in Pauletti and Motta (2017), 9.0-75.0 $\mathrm{g} \mathrm{kg}^{-1}$.

Sufficient copper concentrations derived from boundary line for 'Grand Nain' grown in Ceará ranged from 3.8 to $6.5 \mathrm{mg} \mathrm{kg}^{-1}$ (Table 5); these limits are close to those determined by the modified and original methods of Kenworthy: 4.3-6.0 mg kg-1 (Table 6) and 4.1-5.9 mg $\mathrm{kg}^{-1}$ (Table 7), respectively. Sufficient ranges determined by boundary line and modified method of Kenworthy for 'Grand Nain' grown in Bahia ranged from 5.9 to $6.8 \mathrm{mg}$ $\mathrm{kg}^{-1}$ (Table 5 and 6 ); this range slightly differs from that determined by the original method of Kenworthy: 5.3-6.5 $\mathrm{mg} \mathrm{kg}{ }^{-1}$ (Table 7). Upper limits of the ranges reported herein are close to the lower limit of the range in Quaggio and Raij (1997), 6.0-30.0 $\mathrm{mg} \mathrm{kg}^{-1}$, same range reported by Pauletti and Motta (2017). 
Sufficient iron concentration range derived from boundary line for 'Grand Nain' grown in Ceará was 49.8$70.2 \mathrm{mg} \mathrm{kg}^{-1}$ (Table 5); the upper limit was very close to the limits determined by the modified and original methods of Kenworthy: 58.9-72.0 $\mathrm{mg} \mathrm{kg}^{-1}$ (Table 6) and 54.8-68.7 $\mathrm{mg} \mathrm{kg}^{-1}$ (Table 7), respectively, however, the lower limit was slightly lower. For 'Grand Nain' grown in Bahia, sufficiency range derived from boundary line ranged from 51.5 to $62.6 \mathrm{mg} \mathrm{kg}^{-1}$ (Table 5), which is close to ranges established by the modified and original method of Kenworthy: 49.3-59.0 mg kg-1 (Table 6) and 47.2-59.0 $\mathrm{mg} \mathrm{kg}^{-1}$ (Table 7), respectively. The ranges reported herein are much lower than the range proposed by Quaggio and Raij (1997), 80.0-360.0 mg kg-1 , however, with the lower limits close to that proposed by Pauletti and Motta (2017), $45-360 \mathrm{~g} \mathrm{~kg}^{-1}$.

Sufficient manganese concentration ranges for 'Grand Nain' grown in Ceará were inconsistent, regardless of the method used. $43.2-79.4 \mathrm{mg} \mathrm{kg}^{-1}$ is the range derived from the boundary line (Table 5); 59.6-187.1 $\mathrm{mg} \mathrm{kg}^{-1}$ is the range estimated by the modified method of Kenworthy; and 65.7-168.9 $\mathrm{mg} \mathrm{kg}^{-1}$, by the original method of Kenworthy (Table 7). In Bahia, however, the boundary line-derived sufficient range was $35.5-59.9 \mathrm{mg}$ $\mathrm{kg}^{-1}$ (Table 5), and was similar to that determined by the modified method of Kenworthy: $36.3-60.2 \mathrm{mg} \mathrm{kg}^{-1}$ (Table 6); and different from the original method of Kenworthy: 57.3-73.5 $\mathrm{mg} \mathrm{kg}^{-1}$ (Table 7). These ranges were much lower and narrower than the range reported by Quaggio and Raij (1997), 200-2000 mg kg-1. This broader range might be because the authors had not used a correction factor (k) to narrow the range (ALVES et al., 2019) for highly fluctuating nutrients, such as micronutrients with CV higher than $20 \%$. However, the ranges are closer to the reported by Pauletti and Motta (2017), 80-180 $\mathrm{g} \mathrm{kg}^{-1}$.

Sufficient zinc concentrations estimated by the boundary line approach for 'Grand Nain' grown in Ceará ranged from 13.6 to $17.8 \mathrm{mg} \mathrm{kg}^{-1}$ (Table 5). Similar results were found by the modified and original method of Kenworthy, 13.1-16.8 $\mathrm{mg} \mathrm{kg}^{-1}$ (Table 6) and 12.5-15.8 $\mathrm{mg} \mathrm{kg}^{-1}$ (Table 7), respectively. For 'Grand Nain' grown in Bahia, the sufficient range determined from boundary line ranged from 13.3 to $15.0 \mathrm{mg} \mathrm{kg}^{-1}$ (Table 5). Similar limits were determined by the modified and original methods of Kenworthy: 13.4-14.9 $\mathrm{mg} \mathrm{kg}^{-1}$ (Table 6) and 12.25-15.4 $\mathrm{mg} \mathrm{kg}^{-1}$ (Table 7), respectively. These ranges are lower and narrower than that found by Quaggio and Raij (1997), 20.0-50.0 mg kg-1, however, with the lower limits close to that proposed by Pauletti and Motta (2017), $12.0-50.0 \mathrm{~g} \mathrm{~kg}^{-1}$.
Sufficient leaf concentration ranges for micronutrients are inconsistent with those reported in the literature. This may be explained by the fluctuating nature of micronutrients in the soil, which is affected by $\mathrm{pH}$, organic matter content, clay content, soil source rock, and, specifically for $\mathrm{Fe}$ and $\mathrm{Mn}$, by the reduction potential affecting these elements' availability under anoxia. These factors can interfere with micronutrient uptake by bananas, affecting how much of the element is found in leaf tissues (ABREU et al., 2007).

Sufficient concentration ranges determined by the boundary line approach for N, P, Ca and S are consistent from one site to the other. However, sufficient $\mathrm{K}$ concentration range determined in bananas grown in Ceará (27.2-36.8 $\left.\mathrm{g} \mathrm{kg}^{-1}\right)$ is broader than the range determined in Bahia (24.1-33.2 $\mathrm{g} \mathrm{kg}^{-1}$ ) probably because the soil $\mathrm{K}$ level in Ceará is about 2.5 times higher than in Bahia (Table 1).

Sufficient $\mathrm{Mg}$ concentration range determined for Ceará $\left(2.0-2.6 \mathrm{~g} \mathrm{~kg}^{-1}\right)$ is lower than the range established for Bahia (2.7-3.0 $\left.\mathrm{g} \mathrm{kg}^{-1}\right)$ in spite of the higher soil $\mathrm{Mg}$ level in Ceará $\left(27 \mathrm{mmol}_{\mathrm{c}} \mathrm{dm}^{-3}\right)$ than in Bahia $\left(10 \mathrm{mmol}_{\mathrm{c}}\right.$ $\mathrm{dm}^{-3}$ ) (Table 1). Competitive inhibition might be the reason for that because the leaf $\mathrm{K} / \mathrm{Mg}$ ratio was $4.3: 1$ in Ceará and 3.5:1 in Bahia; therefore, in Ceará, K uptake increased to the detriment of $\mathrm{Mg}$ uptake. Adequate $\mathrm{K} / \mathrm{Mg}$ ratio at flowering stage ranges from $2.5: 1$ to $3.5: 1$. Ratios equal to or higher than 4.0:1 are considered too high (SILVA, 2015), since it indicates Mg deficiency due to excessive $\mathrm{K}$. This might be associated with frequent $\mathrm{K}$ fertilization, seeing that $\mathrm{K} / \mathrm{Mg}$ ratios in the two soils are $0.38: 1$ and 0.34:1 in Ceará and Bahia, respectively, which are within the ideal range 0.2-0.5 (SILVA, 2015). Likewise, the higher soil $\mathrm{Ca}$ level in Ceará raises $\mathrm{Ca} / \mathrm{Mg}$ ratio to $4.2: 1$, ratio above the optimum range, 1.5-3.0 (SILVA, 2015); as a result, $\mathrm{Mg}$ uptake might be hampered and leaf $\mathrm{Mg}$ concentration decreased. In Bahia, however, $\mathrm{Ca} / \mathrm{Mg}$ ratio, $2.7: 1$, is within the optimum range.

Banana has low root cation exchange capacity, so monovalent cations are more readily taken up. Thus, conditions under which stomata closure is more likely to occur, such as high temperatures and low relative humidity (Table 2), affect $\mathrm{Mg}^{++}$uptake as well as other highly mobile elements that are taken up from the soil by plants through mass flow, i.e., the water potential difference between the soil and roots as a result of plant transpiration. Magnesium deficiency impairs banana growth and yield, so this might partially be the reason of the lower average yield (52.35 $\mathrm{Mg} \mathrm{ha}^{-1}$ ) recorded in Ceará compared to the average recorded in Bahia (65.15 $\left.\mathrm{Mg} \mathrm{ha}^{-1}\right)$. Moreover, banana yield reductions can be influenced by non-nutritional factors. In this study, high temperature stood out for having a negative effect on Rubisco activity and on membrane permeability, thereby decreasing carboxylation and photosynthesis rates (ARANTES et al., 2018). 
Proposed micronutrient ranges are not consistent between sites owing to micronutrients' highly fluctuating leaf concentrations, which are influenced by soil $\mathrm{pH}$, organic matter content, clay content, and reduction potential. These interfere with nutrient uptake; hence, different sufficiency ranges.

Sufficient concentration ranges established using the boundary line approach and modified method of Kenworthy were very alike for most nutrients, and, in some instances, they were different from the original method of Kenworthy; therefore, it is necessary to determine reference values when establishing sufficiency ranges by the method of Kenworthy, so that plant nutrient status is more accurately assessed.

Sufficient concentration ranges proposed in this paper are different from those reported by Quaggio and Raij (1997) for bananas belonging to the Cavendish subgroup, the same subgroup to which 'Grand Nain' belongs, and grown in the state of São Paulo. These differences indicate an overall decrease in sufficiency ranges. Analogously, Urano et al. (2007) and Deus et al. (2018) reported narrower normal ranges than those found in the literature; Accordingly, these region-specific methods should be considered more accurate by decreasing soil and climate variation effects, i.e., in accordance with the farm's production capacity. This prevents misleading extrapolations and mistakes when transferring knowledge; simply put, different environments, different managements (RESENDE et al., 2017). Additionally, when estimating ranges for highly fluctuating nutrients by the sufficiency range approach, one must consider a correction factor $(\mathrm{k})$, which is often not employed by authors.

\section{Conclusions}

Potential response curves were determined as to leaf macro- and micronutrient concentrations, balance indices of Kenworthy, with high predictive power, for irrigated 'Grand Nain' bananas.

Sufficiency ranges were established for macro and micronutrient concentrations, balance indices of Kenworthy. These ranges allow improved nutrient status assessment of irrigated 'Grand Nain' bananas.

\section{Acknowledgment}

This work was carried out with the support of the Coordination for the Improvement of Higher Education Personnel - Brazil (CAPES) - Financing Code 001.

Paper extracted from the Masters dissertation of the first author.

\section{References}

ABREU, C.A.; LOPES, A.S.; SANTOS, G.C.G. Micronutrientes. In: NOVAIS, R.F.; ALVAREZ, V.V.H.; BARROS, N.F. de; FONTES, R.L.F.; CANTARUTTI, R.B.; NEVES, J.C.L. (ed.). Fertilidade do solo. Viçosa: Sociedade Brasileira de Ciência do Solo, 2007. p.645736.

ALI, A.M. Nutrient sufficiency ranges in mango using boundary-line approach and compositional nutrient diagnosis norms in El-Salhiya, Egypt. Communications in Soil Science and Plant Analysis, Bansigstoke, v.49, n.2, p.188-201, 2018.

ALMEIDA, E.I.B.; DEUS, J.A.L.; CORREAA, M.C.M.; CRISOSTOMO, L.A.; NEVES J.C.L. Linha de fronteira e chance matemática na determinação do estado nutricional de pitaia. Revista Ciência Agronômica, Fortaleza, v.47, n.4, p. 744-754, 2016.

ALVES, J.F.T.; DONATO, S.L.R.; DONATO P.E.R.; SILVA J.A. da; GUIMARÃES B.V.C. Establishment of sufficiency ranges to determine the nutritional Status of 'Gigante' cactus pear - micronutrients. Journal of Agricultural Science, Cambridge, v.11, n.18, p.222-229, 2019.

ARANTES, A.M.; DONATO S.L.R.; SIQUEIRA D.L.; COELHO E.F. Gas exchange in 'Pome' banana plants grown under different irrigation systems. Engenharia Agrícola, Jaboticabal, v.38, n.2, p.197-207, 2018.

BARKER, A.V.; PILBEAM, D.J. Handbook of plant nutrition. Boca Raton: CRC Press, 773p. 2015.

BATAGLIA, O.C.; FURLANI, A.M.C.; TEIXEIRA, J.P.F.; FURLANI, P.R.; GALLO J.R. Métodos de análise química de plantas. Campinas: IAC. 1993. 48p. (Boletim Técnico, 78)

BLANCO-MACÍAS, F.; MAGALLANES-QUINTANAR, R.; VALDEZ-CEPEDA, R.D.; VÁZQUEZ-ALVARADO, R.; OLIVARES-SÁENZ, E.; GUTIÉRREZ-ORNELAS, E.; MURILLO AMADOR, B. Nutritional reference values for Opuntia ficus indica determined by means of the boundary line approach. Journal of Plant Nutrition and Soil Science, Weinheim, v.173, n.1, p.927-934, 2010.

BORGES, A.L.; SILVA, S. de O.E.; CALDAS, C.R.; LEDO, C.A. da S. Teores foliares de nutrientes em genótipos de bananeira. Revista Brasileira de Fruticultura, Jaboticabal, v.28, n.2, p.314-318, 2006. 
DAMATTO JUNIOR, E.R.; VILLAS BOAS, R.L.; NOMURA, E.S.; FUZITANI, E.J.; GARCIA, V.A. Alterações nos teores nutricionais foliares de bananeira 'prata-anã' adubada com composto orgânico em cinco ciclos de produção. Revista Brasileira de Fruticultura, Jaboticabal, v.33, p.692-698, 2011. Número especial

DEUS, J.A.L.; NEVES, J.C.L.; SOARES, I.; ALVAREZ, V.V.H.; ALBUQUERQUE, F.M.R.; SANTOS, L.L.; NATALE, W. Modeling in the adjustment of fertilization recommendation through leaf analysis in fertigated 'Prata' banana. Revista Brasileira de Ciência do Solo, Viçosa, MG, v.42, p.1-19, 2018.

DONATO, S.L.R.; LÉDO, A.A.; PEREIRA, M.C.T.; COELHO, E.F.; COTRIM C.E. Estado nutricional de bananeiras tipo prata sob diferentes sistemas de irrigação. Pesquisa Agropecuária Brasileira, Brasília, DF, v.45, n.9, p. 980-988, 2010.

HOFFMANN, R.B.; OLIVEIRA, F.H.T.; SOUZA, A.P.; GHEYI, H.R.; SOUZA JÚNIOR, R.F. Acúmulo de matéria seca e de macronutrientes em cultivares de bananeira irrigada. Revista Brasileira de Fruticultura, Jaboticabal, v.32, n.1, p.268-275, 2010.

IHESHIULO, E.M.A.; ABBEY, L.; HAMMERMEISTER, A.M. Nutrient sufficiency levels for haskap (Lonicera caerulea L.) using the boundary-line approach. Canadian Journal Plant Science, Ontario, v.1, n.1, p. 1-29, 2019.

IUSS Working Group WRB. World reference base for soil resources 2014, update 2015. International soil classification system for naming soils and creating legends for soil maps. Rome: FAO, 2015. (World Soil Resources Reports, 106).

KENWORTHY, A.L. Interpreting the balance of nutrientelements in leaves of fruit trees. In: REUTHER, W. (ed.). Plant analysis and fertilizers problems. Washington: American Institute of Biological Science, 1961. p.28-43.

LAFOND, J. Optimum leaf nutrient concentrations of wild lowbush blueberry in Quebec. Canadian Journal of Plant Science, Ottawa, v.1, n.1, p.341-347, 2009.

LIMA NETO, A.J.; NEVES J.C.L.; MARTINEZ H.E.P.; SOUSA J.S.; FERNANDES L.V. Establishment of critical nutrient levels in soil and plant for eucalyptus. Revista Brasileira de Ciência do Solo, Viçosa, MG, v.44, n.e0190150, p.1-16, 2020.

MAIA, C.E.; MORAIS, E.R.C. Boundary line model to estimate the nutrient sufficiency range in muskmelon leaves. Revista Brasileira de Ciência do Solo, Viçosa, MG, v.40, n.e0160033, p.1-8, 2016.
MARSCHNER, H. Marschner's mineral nutrition of higher plants. San Diego: Academic Press, 2012. 672 p.

MARTIN-PRÉVEL, P.; BANANA. IN: MARTINPREVEL, P.; GAGNARD, J.; GAUTIER P. Plant analysis: as a guide to the nutrient requirements of temperate and tropical crops. Paris: Lavoisier Publising, 1987.

MCCRAY, J.M.; JI, S.; POWELL, G.; MONTES, G.; PERDOMO, R.; LUO, Y. Boundary lines used to determine sugarcane production limits at leaf nutrient concentrations less than optimum. Communications in Soil Science and Plant Analysis, Bansigstoke, v.41, n.5, p.606-622, 2010.

NJUKENG, J.N.; EHABE, E.E.; NKENG, G.E.; SCHICK, J.; KRATZ, S.; SCHNUG, E. Investigations on the nutritional status of Hevea brasiliensis plantations in the humid forest zone of Cameroon. Part 2: Establishment of macro nutrient norms. Journal für Kulturpflanzen, Stuttgart, v.65, n.10, p. 376-384, 2013.

PAULETTI, V.; MOTTA, A.C.V. Manual de adubação e calagem para o estado do Paraná. Curitiba: SBCS/ NESPAR, 2017. 482p.

PULITO, A.P.; GONÇALVES, J.L.M.; SMETHURST, P.J.; ARTHUR JUNIOR, J.C.; ALCARDE ALVARES, C.; ROCHA, J.H.T.; HUBNER, A.; MORAES, L.F.; MIRANDA, A.C.; KAMOGAWA, M.Y.; GAVA, J.L.; CHAVES, R. Available nitrogen and responses to nitrogen fertilizer in Brazilian eucalypt plantations on soils of contrasting texture. Forests, Basel, v.6, n.4, p. 973-991, 2015.

QUAGGIO, J.A.; RAIJ, B.V. Frutíferas. In: RAIJ, B.V. et al. (ed.) Recomendações de adubação e calagem para o Estado de São Paulo. 2.ed. rev. Campinas: IAC, 1997. p.121-153. (Boletim Técnico, 100).

RAGHUPATHI, H.B.; REDDY, B.M.C.; SRINIVAS, K. Multivariate diagnosis of nutrient imbalance in banana, Communications in Soil Science and Plant Analysis, Bansigstoke, v.33, n.13/14, p. 2131-2143, 2002.

RESENDE, M.; CURI, N.; REZENDE, S.B. Uso das informações pedológicas agrícola e não agrícola. In: CURI N., KER J.C., NOVAIS R.F., VIDAL-TORRADO P., SCHAEFER C.E.G.R. (ed.). Pedologia - solos dos biomas brasileiros. Viçosa (MG): Sociedade Brasileira de Ciência do Solo, 2017. p.47-110. 
RODRIGUES, M.G.V.; PACHECO, D.D.; NATALE, W.; SILVA, J.T.A.D. Amostragem foliar da bananeira 'PrataAnã'. Revista Brasileira de Fruticultura, Jaboticabal, v.32, n.1, p. 321-325, 2010.

SANTOS, H. G.; JACOMINE, P. K. T.; ANJOS, L. H. C.; OLIVEIRA, V. A.; LUMBRERAS, J. F.; COELHO, M. R.; ALMEIDA, J. A.; ARAÚJO FILHO, J. C.; OLIVEIRA, J. B.; CUNHA, T. J. F. Sistema brasileiro de classificação de solos. 5. ed. rev. ampl. Brasília: Embrapa, 2018. 356p.

SILVA, J.T.A. Solo, adubação e nutrição para bananeira. Informe Agropecuário, Belo Horizonte, v.36, n.288, p.74-83, 2015.

SILVA, J.T.A.; CARVALHO, J.G. Estabelecimento de normas DRIS para bananeira 'Prata-Anã' (AAB) sob irrigação. Ciência e Agrotecnologia, Lavras, v.30, n.1, p. 43-51, 2006.

SILVA, J.T.A.; CARVALHO, J.G. Propriedades do solo, estado nutricional e produtividade de bananeira 'PrataAnã' (AAB) irrigadas com águas calcárias. Ciência e Agrotecnologia, Lavras, v.28, n.2, p. 332-338, 2004.
SILVA, J.T.A.; PEREIRA, R.D.; RODRIGUES M.G. Adubação da bananeira 'Prata Anã' com diferentes doses e fontes de nitrogênio. Revista Brasileira de Engenharia Agrícola e Ambiental, Campina Grande, v.16, n.12, p. 1314-1320, 2012.

SILVA, J.T.A.; RODRIGUES, M.G.V. Produção da bananeira 'Prata Anã' em função da aplicação de adubo fosfatado, em quatro ciclos. Pesquisa Agropecuária Brasileira, Brasília, DF, v.48, n.6, p.613-618, 2013.

SOTO BALLESTERO, M. Bananos: tecnicas de produccion, poscosecha y comercializacion. Guacimo: EARTH, 2008. CD-ROM

SOIL SURVEY STAFF. Keys to soil taxonomy. $12^{\text {th }}$ ed. Washington: United States Department of Agriculture, Natural Resources Conservation Service, 2014.

URANO, E.O.M.; KURIHARA, C.H.; MAEDA, S.; VITORINO, A.C.T.; GONÇALVES, M.C.; MARCHETTI, M.E. Determinação de teores ótimos de nutrientes em soja pelos métodos chance matemática, sistema integrado de diagnose e recomendação e diagnose da composição nutricional. Revista Brasileira de Ciência do Solo, Viçosa, MG, v.31, n.1, p.63-72, 2007.

WADT, P.G.S.; NOVAIS, R.D.; ALVAREZ, V.V.H.; FONSECA, S.; BARROS N.D. Valores de referência para macronutrientes em eucalipto obtidos pelos métodos DRIS e chance matemática. Revista Brasileira de Ciência do Solo, Viçosa, MG, v.22, n.1, p.685-692, 1998. 